\title{
Finite element formulae for internal forces of Bernoulli-Euler beams under moving vehicles
}

\author{
Ping Lou ${ }^{\mathrm{a}, \mathrm{b}, \mathrm{c}, \mathrm{d}}$ and F. T. K. Au ${ }^{\mathrm{d}, *}$
}

a. School of Civil Engineering, Railway Campus, Central South University, 22 Shao-shan-nan Road, Changsha, Hunan 410075, China.

b. Key Laboratory of Heavy Railway Engineering Structure of Education Ministry, Railway Campus, Central South University, 22 Shao-shan-nan Road, Changsha, Hunan 410075, China.

c. Collaborative Innovation Centre of Rail Safety, 22 Shao-shan-nan Road, Changsha, Hunan 410075, China.

d. Department of Civil Engineering, The University of Hong Kong, Pokfulam Road, Hong Kong, China.

\begin{abstract}
:
This paper presents finite element formulae for calculating accurately the internal forces, namely bending moment and shear force, of Bernoulli-Euler beams under moving vehicles. The formulae for evaluating these internal forces are derived based on the dynamic equilibrium conditions and the solution procedure is also given. The correctness of the proposed formulae is verified by comparing with available closed-form solutions. The internal forces of simply supported and continuous beams subjected to moving vehicles are obtained by several methods. The numerical results show that the proposed formulae are efficient and accurate in predicting the internal forces.
\end{abstract}

\section{Keywords:}

Bending moment; Bernoulli-Euler beam; Finite element; Gibbs phenomenon; Moving load; Shear force

\footnotetext{
${ }^{*}$ Corresponding author at: Department of Civil Engineering, The University of Hong Kong, Pokfulam Road, Hong Kong, China. TEL: +852 28592650; FAX: +852 25595337.

E-mail addresses: pinglou@mail.csu.edu.cn; pingloucsu@126.com (P. Lou), francis.au@hku.hk (F. T. K. Au)
} 


\section{Introduction}

The dynamic analysis of flexible structures under moving loads has been of interest to engineers for many years. The dynamic response of the moving load problem may be determined by modal analysis using a series expansion of the trial solution in terms of the eigenfunctions of the undamped and unloaded continuous system [1-3]. This conventional series expansion method (CSEM) quickly converges to the solution for deflection. However, to obtain the generalized internal forces, such as bending moment and shear force, convergence is very slow as it involves the higher-order derivatives.

To overcome the drawback of CSEM, some researchers used various series expansions to evaluate the internal forces in three classes of problems, i.e. the moving force problem, the moving mass problem, and the moving oscillator or vehicle problem. Frýba [3] introduced two methods to calculate the internal forces in beams, including the integro-differential equation method using the influence functions of internal forces, and the combined method using CSEM in conjunction with integro-differential equations. Pesterev and Bergman [4] proposed an improved series expansion for calculating the internal forces in beams for cases of moving forces and oscillators. In this method, the beam response is evaluated as the sum of the conventional modal series expansion and a correction function, which takes into account approximately the contribution associated with the truncated higher-order eigenfunctions. A further improvement has also been proposed later by Pesterev et al. [5]. Biondi et al. [6] presented two improved expansion methods for calculating the internal forces in beams for the moving mass problem. In the first method, the response is evaluated by considering the particular solution of the differential equation governing the moving mass problem associated with the truncated terms of the eigenfunction expansion. The second one can be considered as an extension of CSEM, in which the eigensolutions of the undamped system are evaluated taking into account the inertial effects of moving masses. Biondi and Muscolino [7] proposed another series expansion method with improved convergence and accuracy for calculating the internal forces in beams taking into account the gravitational, inertial and damping effects due 
to the moving oscillators. Bilello et al. [8] presented a correction procedure to improve the evaluation of the dynamic response of linear, proportionally damped, continuous onedimensional systems traversed by moving loads including a moving force and a moving mass. The method is based on the separation of the low-frequency response evaluated by CSEM and the high-frequency response that is obtained as a series expansion of the particular solution. The above-mentioned researchers have adopted various series expansions to evaluate the internal forces along continuous structures under moving forces, moving masses and moving oscillators, and can capture the discontinuity in the internal forces due to these moving loads. However it is difficult to obtain the eigenfunctions required by CSEM.

The finite element method (FEM) has been used to solve various forms of moving load problems, providing results of displacement, acceleration and bending moment at critical sections of a beam, but little work has addressed the internal force distributions. In modelling internal forces distribution in moving load problems, the accuracy of FEM is often considered inferior to CSEM [7]. Subsequently finite element formulae $[9,10]$ have been presented to calculate the internal forces in beams supported on discrete or continuous foundations under moving forces.

This paper presents finite element formulae for calculating the internal forces, namely bending moment and shear force, at any arbitrary sections (including the element nodes) of a Bernoulli-Euler beam under moving vehicles. The proposed formulae can efficiently capture discontinuities in the variation of shear force along simply supported and continuous beams under moving forces or vehicles.

\section{Modelling of a train-bridge interaction system}

\subsection{Models of vehicle and bridge}

Fig. 1 shows a train comprising a series of identical vehicles running on a multi-span continuous bridge. The train comprises $N_{\mathrm{v}}$ identical vehicles numbered as $1,2, \ldots, N_{\mathrm{v}}$-th from right to left and proceeds at speed $v$ and acceleration $a$ at time $t$ in the longitudinal 
direction. Each vehicle in the train is modelled as a mass-spring-damper system consisting of a car body, two bogie frames, four wheelsets and two-stage suspensions. All the wheelsets are assumed to contact rigidly and continuously with the rails as they roll over. Each vehicle has 6 independent degrees of freedom (DOFs), including the vertical displacement and rotation at the centre of gravity of each of the car body, and rear and front bogie frames. It is assumed that downward vertical displacements and clockwise rotations of vehicle are taken as positive and that they are measured with reference to their respective static equilibrium positions before coming onto the bridge.

In short bridges, the presence of the track structure may increase the overall damping; it may as well reduce the response to some extent due to the distribution of axle loads in the longitudinal direction [11]. Nevertheless, aside from these facts, the effect of the track on the dynamic response of a bridge structure under a moving train is not of great importance [12], and consequently the bridge deck and the track have been modelled as a Bernoulli-Euler beam structure. Downward deflection of beam is taken as positive and it is measured with reference to the vertical static equilibrium positions of the bridge under its permanent loading but in the absence of vehicles. The top surface irregularity of beam is denoted by $r(x)$ in terms of the length abscissa $x$, with downward deviation considered positive. The physical parameters for modelling the vehicle and bridge interaction system are taken from $\mathrm{Wu}$ and Yang [13], and Yang and $\mathrm{Wu}[14]$ as shown in Fig. 2 and summarized in Table 1.

\subsection{Equation of motion for a train-bridge interaction system}

Similar to the train-track-bridge interaction system modelled by Lou [15], one can obtain the equation of motion for a train-bridge interaction system in terms of the acceleration

vectors $\ddot{\mathbf{X}}$, the velocity vectors $\dot{\mathbf{X}}$, the displacement vectors $\mathbf{X}$, the force vectors $\mathbf{F}$, the mass matrices $\mathbf{M}$, the stiffness matrices $\mathbf{K}$ and the damping matrices $\mathbf{C}$ as

$$
\left[\begin{array}{cc}
\mathbf{M}_{\mathrm{v}} & \mathbf{0} \\
\mathbf{0} & \mathbf{M}_{\mathrm{b}}
\end{array}\right]\left\{\begin{array}{l}
\ddot{\mathbf{X}}_{\mathrm{v}} \\
\ddot{\mathbf{X}}_{\mathrm{b}}
\end{array}\right\}+\left[\begin{array}{cc}
\mathbf{C}_{\mathrm{v}} & \mathbf{C}_{\mathrm{vb}} \\
\mathbf{C}_{\mathrm{bv}} & \mathbf{C}_{\mathrm{b}}
\end{array}\right]\left\{\begin{array}{l}
\dot{\mathbf{X}}_{\mathrm{v}} \\
\dot{\mathbf{X}}_{\mathrm{b}}
\end{array}\right\}+\left[\begin{array}{cc}
\mathbf{K}_{\mathrm{v}} & \mathbf{K}_{\mathrm{vb}} \\
\mathbf{K}_{\mathrm{bv}} & \mathbf{K}_{\mathrm{b}}
\end{array}\right]\left\{\begin{array}{l}
\mathbf{X}_{\mathrm{v}} \\
\mathbf{X}_{\mathrm{b}}
\end{array}\right\}=\left\{\begin{array}{l}
\mathbf{F}_{\mathrm{v}} \\
\mathbf{F}_{\mathrm{b}}
\end{array}\right\}
$$


where the subscripts " $\mathrm{v}$ " and "b" denote the vehicle and bridge, respectively, as elaborated below.

\subsubsection{Displacement vectors}

The displacement vector of vehicles $\mathbf{X}_{\mathrm{v}}$ of order $\left(6 \times N_{\mathrm{v}}\right) \times 1$ can be written as

$$
\mathbf{X}_{\mathrm{v}}=\left[\begin{array}{llll}
\mathbf{X}_{\mathrm{v} 1} & \mathbf{X}_{\mathrm{v} 2} & \cdots & \mathbf{X}_{\mathrm{vN} N_{\mathrm{v}}}
\end{array}\right]^{\mathrm{T}}
$$

where the superscript "T" denotes transpose of matrix, and $\mathbf{X}_{\mathrm{vj}}\left(j=1,2, \ldots, N_{\mathrm{v}}\right)$ of order $1 \times 6$ denotes the displacement vector of the $j$-th vehicle given as

$$
\mathbf{X}_{\mathrm{v} j}=\left[\begin{array}{llllll}
y_{\mathrm{c} j} & \theta_{\mathrm{c} j} & y_{\mathrm{t} 1 j} & \theta_{\mathrm{t} 1 j} & y_{\mathrm{t} 2 j} & \theta_{\mathrm{t} 2 j}
\end{array}\right]
$$

The displacement vector of the bridge $\mathbf{X}_{\mathrm{b}}$ of order $N_{\mathrm{b}} \times 1$ can be written as

$$
\mathbf{X}_{\mathrm{b}}=\left[\begin{array}{llll}
q_{\mathrm{b} 1} & q_{\mathrm{b} 2} & \cdots & q_{\mathrm{bN}}
\end{array}\right]^{\mathrm{T}}
$$

where $N_{\mathrm{b}}$ denotes the total number of DOFs of the bridge.

\subsubsection{Matrices of the vehicles}

The mass matrix of vehicles $\mathbf{M}_{\mathrm{v}}$ of order $\left(6 \times N_{\mathrm{v}}\right) \times\left(6 \times N_{\mathrm{v}}\right)$ can be written as

$$
\mathbf{M}_{\mathrm{v}}=\operatorname{diag}\left[\mathbf{M}_{\mathrm{v} 1} \quad \mathbf{M}_{\mathrm{v} 2} \quad \cdots \quad \mathbf{M}_{\mathrm{vN}}\right]
$$

where $\mathbf{M}_{\mathrm{vj}}$ of order $6 \times 6$ denotes the mass matrix of the $j$-th vehicle given as

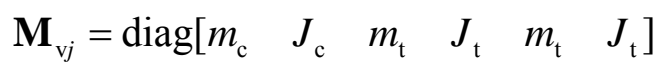

The stiffness matrix of vehicles $\mathbf{K}_{\mathrm{v}}$ of order $\left(6 \times N_{\mathrm{v}}\right) \times\left(6 \times N_{\mathrm{v}}\right)$ can be written as

$$
\mathbf{K}_{\mathrm{v}}=\operatorname{diag}\left[\begin{array}{llll}
\mathbf{K}_{\mathrm{v} 1} & \mathbf{K}_{\mathrm{v} 2} & \cdots & \mathbf{K}_{\mathrm{vN}}
\end{array}\right]
$$

where $\mathbf{K}_{\mathrm{vj}}$ of order $6 \times 6$ denotes the stiffness matrix of the $j$-th vehicle given as

$$
\mathbf{K}_{\mathrm{vj}}=\left[\begin{array}{cccccc}
2 k_{\mathrm{s}} & 0 & -k_{\mathrm{s}} & 0 & -k_{\mathrm{s}} & 0 \\
& 2 k_{\mathrm{s}} L_{\mathrm{c}}{ }^{2} & k_{\mathrm{s}} L_{\mathrm{c}} & 0 & -k_{\mathrm{s}} L_{\mathrm{c}} & 0 \\
& & k_{\mathrm{s}}+2 k_{\mathrm{p}} & 0 & 0 & 0 \\
& & & 2 k_{\mathrm{p}} L_{\mathrm{t}}{ }^{2} & 0 & 0 \\
& & & & k_{\mathrm{s}}+2 k_{\mathrm{p}} & 0 \\
& & & & & 2 k_{\mathrm{p}} L_{\mathrm{t}}{ }^{2}
\end{array}\right]
$$


The damping matrix of vehicles $\mathbf{C}_{\mathrm{v}}$ of order $\left(6 \times N_{\mathrm{v}}\right) \times\left(6 \times N_{\mathrm{v}}\right)$ can be obtained by simply replacing " $k_{\mathrm{s}}$ " and " $k_{\mathrm{p}}$ " in the corresponding stiffness matrix $\mathbf{K}_{\mathrm{v}}$ by " $c_{\mathrm{s}}$ " and " $c_{\mathrm{p}}$ ", respectively.

\subsubsection{Matrices of the bridge}

The cubic Hermitian functions are used as shape functions of the beam element. The mass matrix $\mathbf{M}_{\mathrm{b}}$ of order $N_{\mathrm{b}} \times N_{\mathrm{b}}$ of the bridge can be written as

$$
\mathbf{M}_{\mathrm{b}}=\mathbf{M}_{\mathrm{b} 1}+\mathbf{M}_{\mathrm{b} 2}
$$

with $\quad \mathbf{M}_{\mathrm{b} 2}=\sum_{j=1}^{N_{\mathrm{v}}} \sum_{h=1}^{4} m_{\mathrm{w}} \cdot \mathbf{N}_{h j}^{\mathrm{T}} \cdot \mathbf{N}_{h j}$

$$
\begin{aligned}
& \mathbf{N}_{h j}=\left[\begin{array}{llllllllllll}
0 & 0 & \cdots & 0 & N_{1} & N_{2} & N_{3} & N_{4} & 0 & \cdots & 0 & 0
\end{array}\right]_{\xi=\xi_{h j}} \\
& N_{1}=1-3(\xi / l)^{2}+2(\xi / l)^{3} \\
& N_{3}=3(\xi / l)^{2}-2(\xi / l)^{3}
\end{aligned}
$$

where $\mathbf{M}_{\mathrm{b} 1}$ represents the overall mass matrix of the bridge itself obtained by assembling all its element mass matrices $\int_{0}^{l} \bar{m}_{\mathrm{b}} \mathbf{N}^{\mathrm{T}} \mathbf{N d} \xi$ of order $4 \times 4$, in which $\bar{m}_{\mathrm{b}}$ denotes the mass per unit length of beam, $\xi$ denotes the local coordinate measured from the left node of element that has a total length of $l$, and $\mathbf{N}=\left[\begin{array}{llll}N_{1} & N_{2} & N_{3} & N_{4}\end{array}\right] ; \mathbf{M}_{\mathrm{b} 2}$ represents the overall mass matrix induced by all wheel masses; the solid circles $(\bullet)$ in the beam as shown in Fig. 2 denote the nodes of the elements; each of $\xi_{1 j}, \xi_{2 j}, \xi_{3 j}$ and $\xi_{4 j}$ for wheelsets from right to left of the $j$-th vehicle respectively denotes the distance between the wheelset and the left node of the beam element under the wheelset; and $\mathbf{N}_{h j}$ of order $1 \times N_{\mathrm{b}}$ are the shape function matrices for the beam element which is evaluated at the position of the $h$-th wheelset of the $j$ th vehicle.

The stiffness matrix $\mathbf{K}_{\mathrm{b}}$ of bridge of order $N_{\mathrm{b}} \times N_{\mathrm{b}}$ can be expressed as 


$$
\mathbf{K}_{\mathrm{b}}=\mathbf{K}_{\mathrm{b} 1}+\mathbf{K}_{\mathrm{b} 2}
$$

with $\quad \mathbf{K}_{\mathrm{b} 2}=\sum_{j=1}^{N_{\mathrm{v}}} \sum_{h=1}^{4}\left[k_{\mathrm{p}} \cdot \mathbf{N}_{h j}^{\mathrm{T}} \cdot \mathbf{N}_{h j}+\left(c_{\mathrm{p}} v+m_{\mathrm{w}} a\right) \cdot \mathbf{N}_{h j}^{\mathrm{T}} \cdot \mathbf{N}_{h j}^{\prime}+m_{\mathrm{w}} v^{2} \cdot \mathbf{N}_{h j}^{\mathrm{T}} \cdot \mathbf{N}_{h j}^{\prime \prime}\right]$

where $\mathbf{K}_{\mathrm{b} 1}$ represents the overall stiffness matrix of the bridge itself obtained by assembling all its element stiffness matrices $\int_{0}^{l} E_{\mathrm{b}} I_{\mathrm{b}} \mathbf{N}^{\prime \prime} \mathbf{N}^{\prime \prime} \mathrm{d} \xi$ of order $4 \times 4$, in which $E_{\mathrm{b}}$ denotes Young's modulus of beam, $I_{\mathrm{b}}$ denotes moment of inertia of beam, and the prime denotes differentiation with respect to the local coordinate $\xi$; and $\mathbf{K}_{\mathrm{b} 2}$ represents the overall stiffness matrix induced by all vehicles.

Similarly, the damping matrix of bridge $\mathbf{C}_{\mathrm{b}}$ of order $N_{\mathrm{b}} \times N_{\mathrm{b}}$ can be written as

$$
\mathrm{C}_{\mathrm{b}}=\mathrm{C}_{\mathrm{b} 1}+\mathrm{C}_{\mathrm{b} 2}
$$

with $\quad \mathbf{C}_{\mathrm{b} 1}=\alpha \cdot \mathbf{M}_{\mathrm{b} 1}+\beta \cdot \mathbf{K}_{\mathrm{b} 2}$

$$
\begin{aligned}
& \alpha=2 \zeta \omega_{1} \omega_{2} /\left(\omega_{1}+\omega_{2}\right) \\
& \beta=2 \zeta /\left(\omega_{1}+\omega_{2}\right) \\
& \mathbf{C}_{\mathrm{b} 2}=\sum_{j=1}^{N_{\mathrm{v}}} \sum_{h=1}^{4}\left(c_{\mathrm{p}} \cdot \mathbf{N}_{h j}^{\mathrm{T}} \cdot \mathbf{N}_{h j}+2 m_{\mathrm{w}} v \cdot \mathbf{N}_{h j}^{\mathrm{T}} \cdot \mathbf{N}_{h j}^{\prime}\right)
\end{aligned}
$$

where $\mathbf{C}_{\mathrm{b} 1}$ represents the overall damping matrix of the bridge itself, which is constructed based on Rayleigh damping using the constants $\alpha$ and $\beta$ obtained from the damping ratio $\zeta$ and the first two natural circular frequencies of the bridge $\omega_{1}$ and $\omega_{2}[13,16]$; and $\mathbf{C}_{\mathrm{b} 2}$ represents the overall damping matrix induced by all vehicles.

\subsubsection{Matrices to account for vehicle-bridge interaction}

The stiffness matrices $\mathbf{K}_{\mathrm{vb}}$ of order $\left(6 \times N_{\mathrm{v}}\right) \times N_{\mathrm{b}}$ and $\mathbf{K}_{\mathrm{bv}}$ of order $N_{\mathrm{b}} \times\left(6 \times N_{\mathrm{v}}\right)$, and damping matrices $\mathbf{C}_{\mathrm{vb}}$ of order $\left(6 \times N_{\mathrm{v}}\right) \times N_{\mathrm{b}}$ and $\mathbf{C}_{\mathrm{bv}}$ of order $N_{\mathrm{b}} \times\left(6 \times N_{\mathrm{v}}\right)$ induced by the interaction between the vehicles and the bridge can be written, respectively, as 


$$
\begin{aligned}
& \mathbf{K}_{\mathrm{vb}}=\sum_{j=1}^{N_{\mathrm{v}}} \mathbf{K}_{\mathrm{v}_{j} \mathrm{~b}_{1}}+\sum_{j=1}^{N_{\mathrm{v}}} \mathbf{K}_{\mathrm{v}_{j} \mathrm{~b}_{2}}+\sum_{j=1}^{N_{\mathrm{v}}} \mathbf{K}_{\mathrm{v}_{j} \mathrm{~b}_{3}}+\sum_{j=1}^{N_{\mathrm{v}}} \mathbf{K}_{\mathrm{v}_{j} \mathrm{~b}_{4}} \\
& \mathbf{K}_{\mathrm{bv}}=\sum_{j=1}^{N_{\mathrm{v}}} \mathbf{K}_{\mathrm{b}_{1} \mathrm{v}_{j}}+\sum_{j=1}^{N_{\mathrm{v}}} \mathbf{K}_{\mathrm{b}_{2} \mathrm{v}_{j}}+\sum_{j=1}^{N_{\mathrm{v}}} \mathbf{K}_{\mathrm{b}_{3} \mathrm{v}_{j}}+\sum_{j=1}^{N_{\mathrm{v}}} \mathbf{K}_{\mathrm{b}_{4} \mathrm{v}_{j}} \\
& \mathbf{C}_{\mathrm{vb}}=\sum_{j=1}^{N_{\mathrm{v}}} \mathbf{C}_{\mathrm{v}_{j} \mathrm{~b}_{1}}+\sum_{j=1}^{N_{\mathrm{v}}} \mathbf{C}_{\mathrm{v}_{j} \mathrm{~b}_{2}}+\sum_{j=1}^{N_{\mathrm{v}}} \mathbf{C}_{\mathrm{v}_{j} \mathrm{~b}_{3}}+\sum_{j=1}^{N_{\mathrm{v}}} \mathbf{C}_{\mathrm{v}_{j} \mathrm{~b}_{4}} \\
& \mathbf{C}_{\mathrm{bvv}}=\sum_{j=1}^{N_{\mathrm{v}}} \mathbf{C}_{\mathrm{b}_{1} \mathrm{v}_{j}}+\sum_{j=1}^{N_{\mathrm{v}}} \mathbf{C}_{\mathrm{b}_{2} \mathrm{v}_{j}}+\sum_{j=1}^{N_{\mathrm{v}}} \mathbf{C}_{\mathrm{b}_{3} \mathrm{v}_{j}}+\sum_{j=1}^{N_{\mathrm{v}}} \mathbf{C}_{\mathrm{b}_{4} \mathrm{v}_{j}}
\end{aligned}
$$

with $\mathbf{K}_{\mathrm{v}_{j} \mathrm{~b}_{1}}=\left[\begin{array}{c}\mathbf{0} \\ \mathbf{0} \\ \vdots \\ -k_{\mathrm{p}} \mathbf{N}_{1 j}-c_{\mathrm{p}} v \mathbf{N}_{1 j}^{\prime} \\ -k_{\mathrm{p}} L_{\mathrm{t}} \mathbf{N}_{1 j}-c_{\mathrm{p}} L_{\mathrm{t}} v \mathbf{N}_{1 j}^{\prime} \\ \vdots \\ \mathbf{0} \\ \mathbf{0}\end{array}\right]_{\left(6 \times N_{\mathrm{v}}\right) \times N_{\mathrm{b}}} \quad \mathbf{K}_{\mathrm{v}_{j} \mathrm{~b}_{2}}=\left[\begin{array}{c}\mathbf{0} \\ \mathbf{0} \\ \vdots \\ -k_{\mathrm{p}} \mathbf{N}_{2 j}-c_{\mathrm{p}} v \mathbf{N}_{2 j}^{\prime} \\ k_{\mathrm{p}} L_{\mathrm{t}} \mathbf{N}_{2 j}+c_{\mathrm{p}} L_{\mathrm{t}} v \mathbf{N}_{2 j}^{\prime} \\ \vdots \\ \mathbf{0} \\ \mathbf{0}\end{array}\right]_{\left(6 \times N_{\mathrm{v}}\right) \times N_{\mathrm{b}}}$

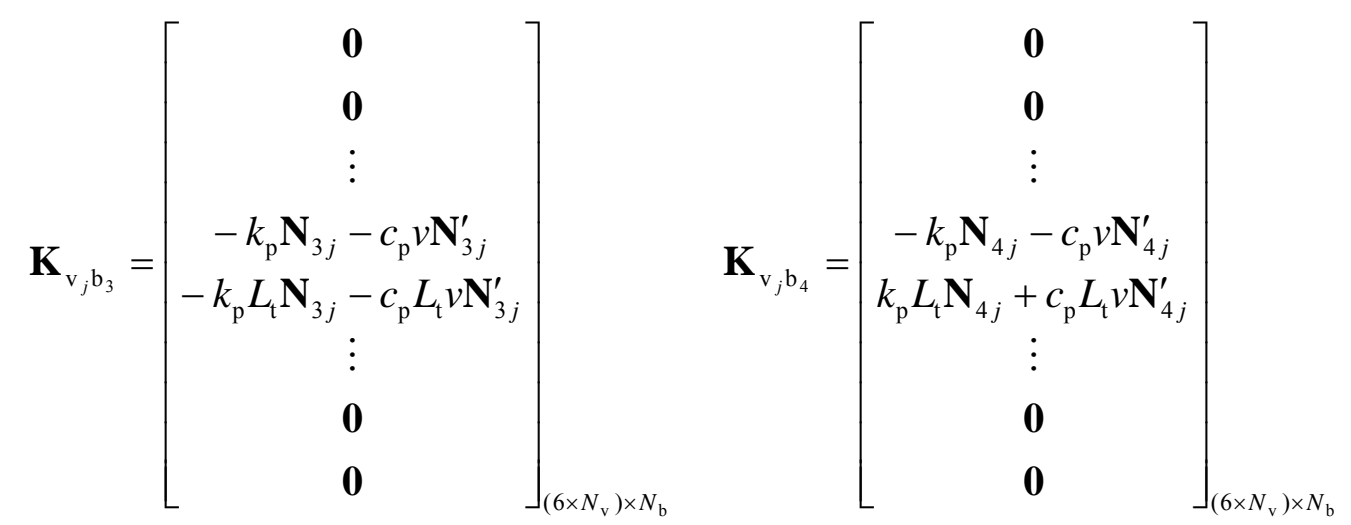

$$
\begin{aligned}
\mathbf{K}_{\mathrm{b}_{1} \mathrm{v}_{j}} & =\left[\begin{array}{lllllllll}
\mathbf{0} & \mathbf{0} & \cdots & -k_{\mathrm{p}} \mathbf{N}_{1 j}^{\mathrm{T}} & -k_{\mathrm{p}} L_{\mathrm{t}} \mathbf{N}_{1 j}^{\mathrm{T}} & \cdots & \mathbf{0} & \mathbf{0}
\end{array}\right]_{N_{\mathrm{b}} \times\left(6 \times N_{\mathrm{v}}\right)} \\
\mathbf{K}_{\mathrm{b}_{2} \mathrm{v}_{j}} & =\left[\begin{array}{lllllllll}
\mathbf{0} & \mathbf{0} & \cdots & -k_{\mathrm{p}} \mathbf{N}_{2 j}^{\mathrm{T}} & k_{\mathrm{p}} L_{\mathrm{t}} \mathbf{N}_{2 j}^{\mathrm{T}} & \cdots & \mathbf{0} & \mathbf{0}
\end{array}\right]_{N_{\mathrm{b}} \times\left(6 \times N_{\mathrm{v}}\right)} \\
\mathbf{K}_{\mathrm{b}_{3} \mathrm{v}_{j}} & =\left[\begin{array}{lllllllll}
\mathbf{0} & \mathbf{0} & \cdots & -k_{\mathrm{p}} \mathbf{N}_{3 j}^{\mathrm{T}} & -k_{\mathrm{p}} L_{\mathrm{t}} \mathbf{N}_{3 j}^{\mathrm{T}} & \cdots & \mathbf{0} & \mathbf{0}
\end{array}\right]_{N_{\mathrm{b}} \times\left(6 \times N_{\mathrm{v}}\right)} \\
\mathbf{K}_{\mathrm{b}_{4} \mathrm{v}_{j}} & =\left[\begin{array}{lllllllll}
\mathbf{0} & \mathbf{0} & \cdots & -k_{\mathrm{p}} \mathbf{N}_{4 j}^{\mathrm{T}} & k_{\mathrm{p}} L_{\mathrm{t}} \mathbf{N}_{4 j}^{\mathrm{T}} & \cdots & \mathbf{0} & \mathbf{0}
\end{array}\right]_{N_{\mathrm{b}} \times\left(6 \times N_{\mathrm{v}}\right)}
\end{aligned}
$$


$\mathbf{C}_{\mathrm{v}_{j} \mathrm{~b}_{1}}=\mathbf{C}_{\mathrm{b}_{1} \mathrm{v}_{j}}^{\mathrm{T}}=\left[\begin{array}{c}\mathbf{0} \\ \mathbf{0} \\ \vdots \\ -c_{\mathrm{p}} \mathbf{N}_{1 j} \\ -c_{\mathrm{p}} L_{\mathrm{t}} \mathbf{N}_{1 j} \\ \vdots \\ \mathbf{0} \\ \mathbf{0}\end{array}\right]_{\left(6 \times N_{\mathrm{v}}\right) \times N_{\mathrm{b}}}$

$\mathbf{C}_{\mathrm{v}_{j} \mathrm{~b}_{2}}=\mathbf{C}_{\mathrm{b}_{2} \mathrm{v}_{j}}^{\mathrm{T}}=\left[\begin{array}{c}\mathbf{0} \\ \mathbf{0} \\ \vdots \\ -c_{\mathrm{p}} \mathbf{N}_{2 j} \\ C_{\mathrm{p}} L_{\mathrm{t}} \mathbf{N}_{2 j} \\ \vdots \\ \mathbf{0} \\ \mathbf{0}\end{array}\right]_{\left(6 \times N_{\mathrm{v}}\right) \times N_{\mathrm{b}}}$

$\mathbf{C}_{\mathrm{v}_{j} \mathrm{~b}_{3}}=\mathbf{C}_{\mathrm{b}_{3} \mathrm{v}_{j}}^{\mathrm{T}}=\left[\begin{array}{c}\mathbf{0} \\ \mathbf{0} \\ \vdots \\ -C_{\mathrm{p}} \mathbf{N}_{3 j} \\ -C_{\mathrm{p}} L_{\mathrm{t}} \mathbf{N}_{3 j} \\ \vdots \\ \mathbf{0} \\ \mathbf{0}\end{array}\right]_{\left(6 \times N_{\mathrm{v}}\right) \times N_{\mathrm{b}}}$

$\mathbf{C}_{\mathrm{v}_{j} \mathrm{~b}_{4}}=\mathbf{C}_{\mathrm{b}_{4} \mathrm{v}_{j}}^{\mathrm{T}}=\left[\begin{array}{c}\mathbf{0} \\ \mathbf{0} \\ \vdots \\ -C_{\mathrm{p}} \mathbf{N}_{4 j} \\ C_{\mathrm{p}} L_{\mathrm{t}} \mathbf{N}_{4 j} \\ \vdots \\ \mathbf{0} \\ \mathbf{0}\end{array}\right]_{\left(6 \times N_{\mathrm{v}}\right) \times N_{\mathrm{b}}}$

where $\mathbf{K}_{\mathrm{v}_{j} \mathrm{~b}_{i}}$ and $\mathbf{K}_{\mathrm{b}_{i} \mathrm{v}_{j}}$ represent the stiffness matrices induced by the interaction between the $i$-th wheelset of the $j$-th vehicle and the bridge, and $\mathbf{C}_{\mathrm{v}_{j} \mathrm{~b}_{i}}$ and $\mathbf{C}_{\mathrm{b}_{i} \mathrm{v}_{j}}$ are the corresponding damping matrices (Fig. 2); $\mathbf{K}_{\mathrm{v}_{j} \mathrm{~b}_{1}}, \mathbf{K}_{\mathrm{v}_{j} \mathrm{~b}_{2}}, \mathbf{C}_{\mathrm{v}_{j} \mathrm{~b}_{1}}$ and $\mathbf{C}_{\mathrm{v}_{j} \mathrm{~b}_{2}}$ consist of zero row vectors except for those corresponding to the two DOFs of front bogie frame of the $j$-th vehicle, while $\mathbf{K}_{\mathrm{b}_{1} \mathrm{v}_{j}}, \mathbf{K}_{\mathrm{b}_{2} \mathrm{v}_{j}}, \mathbf{C}_{\mathrm{b}_{1} \mathrm{v}_{j}}$ and $\mathbf{C}_{\mathrm{b}_{2} \mathrm{v}_{j}}$ consist of similar column vectors. Accordingly, $\mathbf{K}_{\mathrm{v}_{j} \mathrm{~b}_{3}}, \mathbf{K}_{\mathrm{v}_{j} \mathrm{~b}_{4}}, \mathbf{C}_{\mathbf{v}_{j} \mathrm{~b}_{3}}, \mathbf{C}_{\mathrm{v}_{j} \mathrm{~b}_{4}}, \mathbf{K}_{\mathrm{b}_{3} \mathrm{v}_{j}}, \mathbf{K}_{\mathrm{b}_{4} \mathrm{v}_{j}}, \mathbf{C}_{\mathrm{b}_{3} \mathrm{v}_{j}}$ and $\mathbf{C}_{\mathrm{b}_{4} \mathrm{v}_{j}}$ are formed by row or column vectors where the only nonzero elements correspond to the two DOFs of the rear bogie.

\subsubsection{Load vectors of vehicles and bridge}

The load vector $\mathbf{F}_{\mathrm{v}}$ of the vehicles of order $\left(6 \times N_{\mathrm{v}}\right) \times 1$ can be written as

$$
\mathbf{F}_{\mathrm{v}}=\left[\begin{array}{llll}
\mathbf{F}_{\mathrm{v} 1} & \mathbf{F}_{\mathrm{v} 2} & \cdots & \mathbf{F}_{\mathrm{v} N_{\mathrm{v}}}
\end{array}\right]^{\mathrm{T}}
$$

where the load vector of the $j$-th vehicle $\mathbf{F}_{\mathrm{vj}}$ of order $6 \times 1$ is 


$$
\mathbf{F}_{\mathrm{v} j}=\left\{\begin{array}{c}
0 \\
0 \\
k_{\mathrm{p}} r_{\xi=\xi_{1 j}}+k_{\mathrm{p}} r_{\xi=\xi_{2 j}}+c_{\mathrm{p}} v r_{\xi=\xi_{1 j}}^{\prime}+c_{\mathrm{p}} v r_{\xi=\xi_{2 j}}^{\prime} \\
-k_{\mathrm{p}} L_{\mathrm{t}} r_{\xi=\xi_{1 j}}+k_{\mathrm{p}} L_{\mathrm{t}} r_{\xi=\xi_{2 j}}-c_{\mathrm{p}} v L_{\mathrm{t}} r_{\xi=\xi_{1 j}}^{\prime}+c_{\mathrm{p}} v L_{\mathrm{t}} r_{\xi=\xi_{2 j}}^{\prime} \\
k_{\mathrm{p}} r_{\xi=\xi_{3 j}}+k_{\mathrm{p}} r_{\xi=\xi_{4 j}}+c_{\mathrm{p}} v r_{\xi=\xi_{3 j}}^{\prime}+c_{\mathrm{p}} v r_{\xi=\xi_{4 j}}^{\prime} \\
-k_{\mathrm{p}} L_{\mathrm{t}} r_{\xi=\xi_{3 j}}+k_{\mathrm{p}} L_{\mathrm{t}} r_{\xi=\xi_{4 j}}-c_{\mathrm{p}} v L_{\mathrm{t}} r_{\xi=\xi_{3 j}}^{\prime}+c_{\mathrm{p}} v L_{\mathrm{t}} r_{\xi=\xi_{4 j}}^{\prime}
\end{array}\right\}
$$

The load vector of bridge $\mathbf{F}_{\mathrm{b}}$ of order $N_{\mathrm{b}} \times 1$ can be written as

$$
\mathbf{F}_{\mathrm{b}}=\sum_{j=1}^{N_{\mathrm{v}}} \sum_{h=1}^{4} \mathbf{F}_{\mathrm{bhj}}
$$

with

$$
\mathbf{F}_{\mathrm{bhj}}=\left(m_{\mathrm{w}} g+0.5 m_{\mathrm{t}} g+0.25 m_{\mathrm{c}} g-k_{\mathrm{p}} \cdot r_{\xi=\xi_{h j}}-c_{\mathrm{p}} v \cdot r_{\xi=\xi_{h j}}^{\prime}-m_{\mathrm{w}} a \cdot r_{\xi=\xi_{h j}}^{\prime}-m_{\mathrm{w}} v^{2} \cdot r_{\xi=\xi_{h j}}^{\prime \prime}\right) \mathbf{N}_{h j}^{\mathrm{T}}
$$

(for $h=1, \ldots, 4)$

where $\mathbf{F}_{\mathrm{bhj}}$ represents the load vectors induced by the $h$-th wheelset of the $j$-th vehicle acting on the bridge.

After introducing the boundary conditions, Eq. (1) can be solved by time integration such as the Newmark- $\beta$ method or Wilson- $\theta$ method [17] to obtain simultaneously the dynamic responses of train and bridge. Eq. (1) has been written on the assumption that $N_{\mathrm{v}}$ vehicles are acting on the bridge. If certain vehicles are not on the bridge, the corresponding rows and columns in the matrix equation should be deleted.

\section{Formulae for calculating the internal forces at the beam nodes}

At time $t$, the generalized displacement, velocity and acceleration at each beam node can be obtained by time integration from Eq. (1). The forces transmitted to the beam by the moving wheelsets at the contacts can be obtained from

$$
\begin{aligned}
& P_{\mathrm{whj}}=\left(m_{\mathrm{w}} g+0.5 m_{\mathrm{t}}+0.25 \cdot m_{\mathrm{c}}\right) g-m_{\mathrm{w}} \ddot{y}_{\mathrm{whj}} \\
& +c_{\mathrm{p}}\left[\dot{y}_{\mathrm{tsj}}+(-1)^{h} L_{\mathrm{t}} \dot{\theta}_{\mathrm{tsj}}-\dot{y}_{\mathrm{w} h j}\right]+k_{\mathrm{p}}\left[y_{\mathrm{tsj}}+(-1)^{h} L_{\mathrm{t}} \theta_{\mathrm{tsj}}-y_{\mathrm{w} h j}\right] \\
& \quad \text { for } s=1 \text { and } 2 \text {, and } h=2 \mathrm{~s}-1 \text { and } 2 \mathrm{~s}
\end{aligned}
$$

in which 


$$
\begin{array}{cr}
y_{\mathrm{whj}}(t)=\mathbf{N}_{\xi=\xi_{h j}} \cdot \mathbf{q}_{h j}^{\mathrm{e}}+r_{\xi=\xi_{h j}} & \text { for } h=1, \ldots, 4 \\
\dot{y}_{\mathrm{whj}}(t)=\mathbf{N}_{\xi=\xi_{h j}} \cdot \dot{\mathbf{q}}_{h j}^{\mathrm{e}}+v \cdot \mathbf{N}_{\xi=\xi_{h j}}^{\prime} \cdot \mathbf{q}_{h j}^{\mathrm{e}}+v \cdot r_{\xi=\xi_{h j}}^{\prime} & \text { for } h=1, \ldots, 4 \\
\ddot{y}_{\mathrm{whj}}(t)=\mathbf{N}_{\xi=\xi_{h j}} \cdot \ddot{\mathbf{q}}_{h j}^{\mathrm{e}}+2 v \cdot \mathbf{N}_{\xi=\xi_{h j}}^{\prime} \cdot \dot{\mathbf{q}}_{h j}^{\mathrm{e}}+a \cdot \mathbf{N}_{\xi=\xi_{h j}}^{\prime} \cdot \mathbf{q}_{h j}^{\mathrm{e}} & \\
+v^{2} \cdot \mathbf{N}_{\xi=\xi_{h j}}^{\prime \prime} \cdot \mathbf{q}_{h j}^{\mathrm{e}}+a \cdot r_{\xi=\xi_{h j}}^{\prime}+v^{2} \cdot r_{\xi=\xi_{h j}}^{\prime \prime} & \text { for } h=1, \ldots, 4
\end{array}
$$

where $\mathbf{q}_{h j}^{\mathrm{e}}$ denotes the generalized displacement vector of the beam element under the $h$-th wheelset of the $j$-th vehicle.

From the dynamic equilibrium of each beam element [18], the nodal element force vector $\mathbf{f}^{\mathrm{e}}$ may be obtained from the inertial force vector $\mathbf{m}_{\mathrm{b}} \ddot{\mathbf{q}}^{\mathrm{e}}$, the damping force vector $\mathbf{c}_{\mathrm{b}} \dot{\mathbf{q}}^{\mathrm{e}}$, the elastic force vector $\mathbf{k}_{\mathrm{b}} \mathbf{q}^{\mathrm{e}}$, and the equivalent nodal force vector $\mathbf{f}_{\mathrm{E}}$ as

$$
\mathbf{f}^{\mathrm{e}}=\mathbf{m}_{\mathrm{b}} \ddot{\mathbf{q}}^{\mathrm{e}}+\mathbf{c}_{\mathrm{b}} \dot{\mathbf{q}}^{\mathrm{e}}+\mathbf{k}_{\mathrm{b}} \mathbf{q}^{\mathrm{e}}-\mathbf{f}_{\mathrm{E}}
$$

where $\mathbf{m}_{\mathrm{b}}, \mathbf{c}_{\mathrm{b}}$ and $\mathbf{k}_{\mathrm{b}}$ are the element mass, damping and stiffness matrices, respectively; the element damping matrix is similarly taken as $\mathbf{c}_{\mathrm{b}}=\alpha \cdot \mathbf{m}_{\mathrm{b}}+\beta \cdot \mathbf{k}_{\mathrm{b}}$ based on Rayleigh damping; $\ddot{\mathbf{q}}^{\mathrm{e}}, \dot{\mathbf{q}}^{\mathrm{e}}$ and $\mathbf{q}^{\mathrm{e}}$ denote the acceleration, velocity and displacement vectors respectively at the element nodes; and the equivalent nodal force vector $\mathbf{f}_{\mathrm{E}}$ results from all forces acting on the beam element. The nodal element force $\mathbf{f}^{\mathrm{e}}$ can be expressed as $\mathbf{f}^{\mathrm{e}}=\left[\begin{array}{llll}Q_{1}^{\mathrm{e}} & M_{1}^{\mathrm{e}} & Q_{\mathrm{r}}^{\mathrm{e}} & M_{\mathrm{r}}^{\mathrm{e}}\end{array}\right]^{\mathrm{T}}$ as shown in Fig. 3.

In the conventional FEM, the nodal element forces and moments can be calculated based on the higher derivatives of displacement at the nodes as

$$
\mathbf{f}^{\mathrm{e}}=\frac{E_{\mathrm{b}} I_{\mathrm{b}}}{l^{3}}\left[\begin{array}{cccc}
12 & 6 l & -12 & 6 l \\
6 l & 4 l^{2} & -6 l & 2 l^{2} \\
-12 & -6 l & 12 & -6 l \\
6 l & 2 l^{2} & -6 l & 4 l^{2}
\end{array}\right] \mathbf{q}^{\mathrm{e}}=\mathbf{k}_{\mathrm{b}} \mathbf{q}^{\mathrm{e}}
$$

Eq. (21) only accounts for the third term on the right hand side of Eq. (20) and neglects the inertial force, the damping force and the equivalent nodal forces. This is why FEM has been 
considered inaccurate [7] in the evaluation of internal forces along a beam under moving loads.

\section{Formulae for calculating the internal forces at arbitrary sections of beam}

If one is interested in the internal forces along a beam, it is unrealistic to use Eq. (20) direct, as very short beam elements must be adopted with dramatic increase in problem size. It is desirable to develop a method for calculating the internal forces at arbitrary positions of the beam. Fig. 4 shows point A at an arbitrary section of the beam between two adjacent nodes, such that there are $\bar{h}$ number of forces acting between the left node of the element and point A at time $t$. From dynamic equilibrium of the free body shown in Fig. 4, the shear force $Q_{\mathrm{A}}$ and bending moment $M_{\mathrm{A}}$ at point A can be obtained as

$$
\begin{aligned}
& Q_{\mathrm{A}}=\int_{0}^{\xi_{\mathrm{A}}} \bar{m}_{\mathrm{b}} \mathbf{N} \ddot{\mathbf{q}}^{\mathrm{e}} \mathrm{d} \xi-Q_{1}^{\mathrm{e}}-\sum_{s=1}^{\bar{h}} P_{s} \\
& M_{\mathrm{A}}=-Q_{1}^{\mathrm{e}} \xi_{\mathrm{A}}-\sum_{s=1}^{\bar{h}} P_{s}\left(\xi_{\mathrm{A}}-\xi_{s}\right)+M_{1}^{\mathrm{e}}+\int_{0}^{\xi_{\mathrm{A}}}\left(\xi_{\mathrm{A}}-\xi\right) \bar{m}_{\mathrm{b}} \mathbf{N} \ddot{\mathbf{q}}^{\mathrm{e}} \mathrm{d} \xi
\end{aligned}
$$

where $\xi_{s}$ denotes the distance between the left node of the element and the s-th force $P_{\mathrm{s}}, \xi_{\mathrm{A}}$ denotes the distance between the left node of the element and point $\mathrm{A}$, and $Q_{1}^{\mathrm{e}}$ and $M_{1}^{\mathrm{e}}$ can be obtained from Eq. (20). As material damping is internal [19] and it has been modelled separately, no damping force appears in Eqs. (22-a) and (22-b).

If $\bar{m}_{\mathrm{b}}$ is constant, Eqs. (22-a) and (22-b) can be written, respectively, as

$$
\begin{aligned}
& Q_{A}=\bar{m}_{b} \overline{\mathbf{N}}_{\xi=\xi_{\mathrm{A}}} \ddot{\mathbf{q}}^{e}-Q_{l}^{e}-\sum_{s=1}^{\bar{h}} P_{s} \\
& M_{\mathrm{A}}=-Q_{1}^{\mathrm{e}} \xi_{\mathrm{A}}-\sum_{s=1}^{\bar{h}} P_{s}\left(\xi_{\mathrm{A}}-\xi_{s}\right)+M_{1}^{\mathrm{e}}+\bar{m}_{\mathrm{b}}\left(\xi_{\mathrm{A}} \overline{\mathbf{N}}_{\xi=\xi_{\mathrm{A}}}-\widetilde{\mathbf{N}}_{\xi=\xi_{\mathrm{A}}}\right) \ddot{\mathbf{q}}^{\mathrm{e}}
\end{aligned}
$$

with $\overline{\mathbf{N}}=\left[\begin{array}{llll}\bar{N}_{1} & \bar{N}_{2} & \bar{N}_{3} & \bar{N}_{4}\end{array}\right]=\int_{0}^{\xi} \mathbf{N} \mathrm{d} \xi=\int_{0}^{\xi}\left[\begin{array}{llll}N_{1} & N_{2} & N_{3} & N_{4}\end{array}\right] \mathrm{d} \xi$ 


$$
\begin{aligned}
& \widetilde{\mathbf{N}}=\left[\begin{array}{llll}
\widetilde{N}_{1} & \widetilde{N}_{2} & \widetilde{N}_{3} & \widetilde{N}_{4}
\end{array}\right]=\int_{0}^{\xi} \xi \mathbf{N} \mathrm{d} \xi=\int_{0}^{\xi} \xi\left[\begin{array}{llll}
N_{1} & N_{2} & N_{3} & N_{4}
\end{array}\right] \mathrm{d} \xi \\
& Q_{1}^{\mathrm{e}}=\mathbf{m}_{\mathrm{e} 1} \cdot \ddot{\mathbf{q}}^{\mathrm{e}}+\left(\alpha \cdot \mathbf{m}_{\mathrm{e} 1}+\beta \cdot \mathbf{k}_{\mathrm{e} 1}\right) \cdot \dot{\mathbf{q}}^{\mathrm{e}}+\mathbf{k}_{\mathrm{e} 1} \cdot \mathbf{q}^{\mathrm{e}}-\mathbf{f}_{\mathrm{E}, 1} \\
& M_{1}^{\mathrm{e}}=\mathbf{m}_{\mathrm{e} 2} \cdot \ddot{\mathbf{q}}^{\mathrm{e}}+\left(\alpha \cdot \mathbf{m}_{\mathrm{e} 2}+\beta \cdot \mathbf{k}_{\mathrm{e} 2}\right) \cdot \dot{\mathbf{q}}^{\mathrm{e}}+\mathbf{k}_{\mathrm{e} 2} \cdot \mathbf{q}^{\mathrm{e}}-\mathbf{f}_{\mathrm{E}, 2} \\
& \mathbf{m}_{\mathrm{e} 1}=\left[\begin{array}{llll}
\frac{\bar{m}_{\mathrm{b}} l}{420} & \frac{22 \bar{m}_{\mathrm{b}} l^{2}}{420} & \frac{54 \bar{m}_{\mathrm{b}} l}{420} & \frac{-13 \bar{m}_{\mathrm{b}} l^{2}}{420}
\end{array}\right] \\
& \mathbf{k}_{\mathrm{el}}=\left[\begin{array}{llll}
\frac{12 E_{\mathrm{b}} I_{\mathrm{b}}}{l^{3}} & \frac{6 E_{\mathrm{b}} I_{\mathrm{b}}}{l^{2}} & \frac{-12 E_{\mathrm{b}} I_{\mathrm{b}}}{l^{3}} & \frac{6 E_{\mathrm{b}} I_{\mathrm{b}}}{l^{2}}
\end{array}\right] \\
& \mathbf{m}_{\mathrm{e} 2}=\left[\begin{array}{llll}
\frac{\bar{m}_{\mathrm{b}} l}{420} & \frac{22 \bar{m}_{\mathrm{b}} l^{2}}{420} & \frac{54 \bar{m}_{\mathrm{b}} l}{420} & \frac{-13 \bar{m}_{\mathrm{b}} l^{2}}{420}
\end{array}\right] \\
& \mathbf{k}_{\mathrm{e} 2}=\left[\begin{array}{llll}
\frac{6 E_{\mathrm{b}} I_{\mathrm{b}}}{l^{2}} & \frac{4 E_{\mathrm{b}} I_{\mathrm{b}}}{l} & \frac{-6 E_{\mathrm{b}} I_{\mathrm{b}}}{l^{2}} & \frac{2 E_{\mathrm{b}} I_{\mathrm{b}}}{l}
\end{array}\right]
\end{aligned}
$$

where $\mathbf{f}_{\mathrm{E}, 1}$ and $\mathbf{f}_{\mathrm{E}, 2}$ are the equivalent nodal force and moment at left node of beam element, respectively.

Eqs. (22) and (23) can give the internal forces at any arbitrary section in a beam element including both nodes, while Eq. (20) can only give the internal forces at the nodes.

The conventional finite element formulae for calculating the shear force and bending moment at point A can be expressed as

$$
\begin{aligned}
& Q_{\mathrm{A}}=-\left.E_{\mathrm{b}} I_{\mathrm{b}} \frac{\partial^{3} y(\xi, t)}{\partial \xi^{3}}\right|_{\xi=\xi_{\mathrm{A}}}=-\left.E_{\mathrm{b}} I_{\mathrm{b}} \mathbf{N}^{\prime \prime \prime}\right|_{\xi=\xi_{\mathrm{A}}} \mathbf{q}^{\mathrm{e}} \\
& =E_{\mathrm{b}} I_{\mathrm{b}}\left[\frac{-12}{l^{3}} \frac{-6}{l^{2}} \frac{12}{l^{3}} \frac{-6}{l^{2}}\right] \mathbf{q}^{\mathrm{e}} \\
& M_{\mathrm{A}}=-\left.E_{\mathrm{b}} I_{\mathrm{b}} \frac{\partial^{2} y(\xi, t)}{\partial \xi^{2}}\right|_{\xi=\xi_{\mathrm{A}}}=-\left.E_{\mathrm{b}} I_{\mathrm{b}} \mathbf{N}^{\prime \prime}\right|_{\xi=\xi_{\mathrm{A}}} \mathbf{q}^{\mathrm{e}} \\
& =-E_{\mathrm{b}} I_{\mathrm{b}}\left[\frac{12}{l^{3}} \frac{6}{l^{2}} \frac{-12}{l^{3}} \frac{6}{l^{2}}\right] \cdot \xi_{\mathrm{A}} \cdot \mathbf{q}^{\mathrm{e}}+E_{\mathrm{b}} I_{\mathrm{b}}\left[\frac{6}{l^{2}} \frac{4}{l} \frac{-6}{l^{2}} \frac{\frac{2}{l}}{l}\right] \cdot \mathbf{q}^{\mathrm{e}}
\end{aligned}
$$

Eq. (21) is a special case of Eqs. (26-a) and (26-b), which are consistent with the lower half of Eq. (21) for $\xi_{\mathrm{A}}=l$. 
Compared with the proposed formulae in Eqs. (22-a) and (22-b), Eq. (26-a) only considers part of the second term $Q_{1}^{\mathrm{e}}$ on the right hand side of Eq. (22-a), and Eq. (26-b) only considers part of the first term $Q_{1}^{\mathrm{e}} \xi_{\mathrm{A}}$ and the third term $M_{1}^{\mathrm{e}}$ on the right hand side of Eq. (22-b). Therefore compared with Eqs. (20), (22-a) and (22-b), Eq. (26-a) neglects the first and third terms on the right hand side of Eq. (22-a), Eq. (26-b) neglects the second and fourth terms on the right hand side of Eq. (22-b), and both Eq. (26-a) and Eq. (26-b) neglect the first, second and fourth terms on the right hand side of Eq. (20).

\section{Solution procedure}

The solution procedures for efficiently evaluating the internal force distribution along a beam under moving vehicles are as follows.

(a) Obtain the displacement, velocity and acceleration of each DOF of vehicles and beam at time $t$ by time integration from Eq. (1).

(b) Calculate the forces $P_{\text {whj }}$ transmitted to the beam by the moving wheelsets at the contacts using Eq. (19). They will become the force $P_{\mathrm{s}}$ in Eqs. (22) and (23).

(c) For any arbitrary position $x$ along the beam and at time $t$, first calculate the force $Q_{1}^{\mathrm{e}}$ and moment $M_{1}^{\mathrm{e}}$ at the left node of the beam element that contains position $x$ by Eq. (20). Then calculate the shear force $Q(x, t)$ and bending moment $M(x, t)$ by Eqs. (22) and (23) for $x=0, \Delta x, 2 \Delta x, \ldots, L$, where $\Delta x$ is a suitable length increment and $L$ is the length of beam.

\section{Numerical examples}

Three numerical examples are presented to illustrate the application of the proposed formulae in the evaluation of internal forces of beams under moving loads.

Unless otherwise stated, the following assumptions are made:

(a) The beam surface is smooth; 
(b) The damping of beam is neglected;

(c) The loads are moving at constant velocity;

(d) The problems are solved by the Wilson- $\theta$ method with $\theta=1.4$, and the length covered by moving force or vehicle in each time step $\Delta t$ is $0.01 \mathrm{~m}$; and

(e) The interval between adjacent sections for the evaluation of internal force distributions along the beam is also taken to be $\Delta x=0.01 \mathrm{~m}$.

\subsection{Example 1. A simply supported beam under a moving constant force}

Consider a simply supported beam with a single span $L$ of $20 \mathrm{~m}$, a moment of inertia $I_{\mathrm{b}}$ of $3.81 \mathrm{~m}^{4}$, Young's modulus $E_{\mathrm{b}}$ of $29430 \mathrm{MPa}$ and a unit mass $\bar{m}_{\mathrm{b}}$ of $34,088 \mathrm{~kg} / \mathrm{m}$, of which the fundamental frequency is $\omega_{1}=44.75 \mathrm{rad} / \mathrm{s}$. A concentrated force with magnitude $P$ $=215.6 \mathrm{kN}$ runs over the beam from left to right. When the force is at the left end of the beam (i.e. $t=0$ ), the beam is at rest. The velocity parameter $\psi$ is defined as $\psi=v \pi / \omega_{1} L$ for presentation of results.

Figs. 5-7 plot the dynamic magnification factors $D_{\mathrm{M} 1}, D_{\mathrm{M} 2}$ and $D_{\mathrm{M} 3}$ for bending moment obtained by the proposed formula, i.e. Eq. (22-b) or (23-b), and the conventional finite element formula, i.e. Eq. (26-b), with 4 and 8 beam elements of equal lengths against the velocity parameter $\psi$, respectively, which are defined as

$$
D_{\mathrm{M} 1}=M_{1} / M_{\mathrm{st}}, \quad D_{\mathrm{M} 2}=M_{2} / M_{\mathrm{st}}, \quad D_{\mathrm{M} 3}=M_{3} / M_{\mathrm{st}}
$$

where $M_{1}$ denotes the maximum dynamic bending moment of all beam sections for velocity parameter $\psi, M_{2}$ denotes the maximum dynamic bending moment of the beam section beneath the moving force for velocity parameter $\psi, M_{3}$ denotes the maximum dynamic bending moment of the mid-span beam section for velocity parameter $\psi$, and $M_{\text {st }}$ is the static bending moment of the mid-span beam section for a force $P$ acting there (i.e. $\left.M_{\mathrm{st}}=P L / 4\right)$. 
The closed-form solutions for $D_{\mathrm{M} 1}, D_{\mathrm{M} 2}$ and $D_{\mathrm{M} 3}$ using the first 7 eigenfunctions in the series expression are also plotted in Figs. 5-7, respectively. The closed-form solutions are based on the formula given by Warburton [20], namely

$$
\frac{M(x, t)}{M_{\mathrm{st}}}=4\left(1-\frac{v t}{L}\right) \frac{x}{L}-4\left\{\frac{x}{L}-\frac{v t}{L}\right\}+\frac{8}{\pi^{2}} \sum_{i=1}^{n} \frac{\sin i \pi x / L\left[\sin i \pi v t / L-(i / \psi) \sin \omega_{1} t\right]}{i^{2}\left(i^{2} / \psi^{2}-1\right)}
$$

where the term $\{\cdots\}$ is included only if $x \geq v t$.

Figs. 5-7 show excellent agreement between the present and closed-form solutions. The maximum absolute values of deviations of $D_{\mathrm{M} 1}, D_{\mathrm{M} 2}$ and $D_{\mathrm{M} 3}$ of the present solutions with 8 and 4 elements from the closed-form solutions are $0.81 \%$ and $1.88 \%$, respectively, which shows that the accuracy is insensitive to mesh fineness. However, the maximum absolute values of deviations of $D_{\mathrm{M} 1}, D_{\mathrm{M} 2}$ and $D_{\mathrm{M} 3}$ of the conventional finite element solutions with 8 and 4 elements from the closed-form solutions are $7.69 \%$ and $16.89 \%$, respectively, which shows not only the lower accuracy but also that the accuracy drops when the number of elements decreases.

Fig. 8 compares the dynamic magnification factors $D_{\mathrm{M} 1}, D_{\mathrm{M} 2}$ and $D_{\mathrm{M} 3}$ obtained by the proposed method with 8 elements versus velocity parameter $\psi$. Firstly, the maximum values of both $D_{\mathrm{M} 1}$ and $D_{\mathrm{M} 2}$ are 1.55 , occurring at $\psi=0.525$, while the maximum value of $D_{\mathrm{M} 3}$ is 1.447 , occurring at $\psi=0.36$. As $D_{\mathrm{M} 3}$ never exceeds $D_{\mathrm{M} 1}$ for the same velocity parameter, the use of $D_{\mathrm{M} 3}$ (i.e. based on mid-span) for design is inadequate. Secondly, for the velocity parameter range $\psi \leq 0.66$ (i.e. $v \leq 676.9 \mathrm{~km} / \mathrm{h}$ ) that covers the common velocities, $D_{\mathrm{M} 1}$ and $D_{\mathrm{M} 2}$ are the same, which means that the maximum $M(x, t)$ occurs at the section beneath the moving force, i.e. $x=v t$. If one needs the dynamic magnification factor for bending moment within the velocity parameter range $\psi \leq 0.66$, one may calculate $D_{\mathrm{M} 2}$ instead of $D_{\mathrm{M} 1}$, which will be much faster. 
In the following verification of shear force, except for the velocity of the constant moving force that is taken as $50 \mathrm{~m} / \mathrm{s}$, the other parameters are the same as those in the above verification of bending moment. The distributions of shear force along the beam obtained by the proposed formula, i.e. Eq. (22-a) or (23-a), and the conventional finite element formula, i.e. Eq. (26-a), with 4 elements of equal lengths, and the closed-form solution with $i=1-200$ are plotted in Fig. 9 for the instant when the traversed length of the force is 12 m, i.e. $t=0.24$

s. The shear force from the closed-form solution of Timoshenko et al. [21] with $i=1-200$ is

$$
Q(x, t)=-E_{\mathrm{b}} I_{\mathrm{b}} \frac{\partial^{3} y(x, t)}{\partial x^{3}}
$$

with

$$
\begin{aligned}
& y(x, t)=\frac{2 P L^{3}}{\bar{m}_{b} \pi^{2}} \sum_{i=1}^{\infty} \frac{\sin (i \pi x / L) \sin (i \pi v t / L)}{i^{2}\left(i^{2} \pi^{2} \bar{a}^{2}-v^{2} L^{2}\right)}-\frac{2 P L^{4} v}{\bar{m}_{b} \pi^{3} \bar{a}} \sum_{i=1}^{\infty} \frac{\sin (i \pi x / L) \sin \left(i^{2} \pi^{2} \bar{a} t / L^{2}\right)}{i^{3}\left(i^{2} \pi^{2} \bar{a}^{2}-v^{2} L^{2}\right)} \\
& \bar{a}^{2}=\frac{E_{b} I_{b}}{\bar{m}_{b}}
\end{aligned}
$$

Fig. 9 shows excellent agreement of the shear force distribution between the closed-form solution and the present solution even though only 4 elements have been used. However, the conventional finite element method errs seriously especially with the use of coarse meshes, which further confirms the discussions at the end of Section 4. The high frequency oscillations around discontinuity indicate the presence of Gibbs phenomenon in the closedform solution. This example also illustrates that the proposed formulae are accurate and free from Gibbs oscillations.

\subsection{Example 2. A simply supported beam under a moving four-wheelset vehicle}

Consider a four-wheelset vehicle with two-stage suspension system travelling over a simply supported beam of length $30 \mathrm{~m}$. The parameters of the vehicle and the beam are those in Table 1 unless otherwise stated. The beam is at rest at time $t=0 \mathrm{~s}$ when the front wheelset of the front bogie runs onto the left end of the beam.

For the solution by CSEM, the bending moment $M(x, t)$ and shear force $Q(x, t)$ of the simply supported beam under a moving vehicle are given, respectively, as 


$$
\begin{aligned}
& M(x, t)=-E_{\mathrm{b}} I_{\mathrm{b}} \frac{\partial^{2} y(x, t)}{\partial^{2} x}=\sum_{i=1}^{n}\left[E_{\mathrm{b}} I_{\mathrm{b}} A_{i}(i \pi / L)^{2} \sin (i \pi x / L)\right] \\
& Q(x, t)=-E_{\mathrm{b}} I_{\mathrm{b}} \frac{\partial^{3} y(x, t)}{\partial^{3} x}=\sum_{i=1}^{n}\left[E_{\mathrm{b}} I_{\mathrm{b}} A_{i}(i \pi / L)^{3} \cos (i \pi x / L)\right]
\end{aligned}
$$

where $y(x, t)$ denotes the vertical displacement of beam at position $x$ and time $t$ as given by Biggs [22] as

$$
y(x, t)=\sum_{i=1}^{n} A_{i} \sin (i \pi x / L)
$$

in which $A_{i}$ denoting the amplitude of the $i$-th eigenfunction can be determined by the modal coordinate method of Lou et al. [23] as a function of time.

The case with vehicle velocity of $50 \mathrm{~m} / \mathrm{s}$ is analysed by the proposed formulae, i.e. Eqs. (22) and (23), and the conventional finite element formulae, i.e. Eqs. (26-a) and (26-b), with 4 elements of equal lengths, as well as the CSEM, i.e. Eqs. (30) and (31), with $n=200$ for shear force and $n=50$ for bending moment. From the distributions of shear force and bending moment along the beam shown in Figs. 10 and 11 respectively for the instant when the traversed length of the vehicle is $24 \mathrm{~m}$, i.e. $t=0.48 \mathrm{~s}$, excellent agreement is observed between the present solution and that by CSEM even though only 4 elements are used, while the conventional finite element solution performs fairly as explained before. In addition, to study the convergence of the CSEM, the distributions of shear force and bending moment are, respectively, plotted in Figs. 12 and 13 for $n=10,50$, and 200. Obviously the convergence for the shear force is slower than that for the bending moment.

Fig. 10 shows that, while the proposed formula can capture accurately discontinuities in shear force under the wheelsets at $x=4 \mathrm{~m}, 6.5 \mathrm{~m}, 21.5 \mathrm{~m}$ and $24 \mathrm{~m}$ without any Gibbs oscillations, the conventional finite element formula cannot even capture four discontinuities because of the coarse mesh used. Fig. 12 shows that CSEM with $n=10$ cannot capture discontinuities in shear force, but convergence improves when the number of eigenfunctions used increases. Although discontinuities in shear force can be captured by CSEM with $n=200$, Gibbs phenomenon also occurs. 
The vehicle velocity is varied from $10 \mathrm{~m} / \mathrm{s}$ to $200 \mathrm{~m} / \mathrm{s}$ at $2.5 \mathrm{~m} / \mathrm{s}$ intervals for further analysis. Figs. 14-16 plot, respectively, the dynamic magnification factors $D_{\mathrm{M} 1}, D_{\mathrm{M} 2}$ and $D_{\mathrm{M} 3}$ against the velocity, for bending moments obtained by three different procedures: the proposed formula, i.e. Eqs. (22-b) or (23-b), the conventional finite element formula, i.e. Eq. (26-b), with 4 and 8 beam elements of equal lengths, and the CSEM, i.e. Eq. (30) with $n=50$. To apply the definitions of $D_{\mathrm{M} 1}, D_{\mathrm{M} 2}$ and $D_{\mathrm{M} 3}$ in Eq. (27) here, $M_{2}$ is taken as the maximum dynamic bending moment of the sections beneath the four wheelsets, while $M_{\text {st }}$ is taken as the maximum static bending moment of the mid-span section caused by the moving 4-axle vehicle. Figs. 14-16 show excellent agreement between the present solutions just using 4 elements and the solutions by CSEM with $n=50$. However significant differences are observed between the conventional finite element solutions even using 8 elements and the solutions by CSEM with $n=50$. The accuracy and convergence of the present solution and the CSEM are further studied by evaluating the dynamic magnification factor $D_{\mathrm{M} 1}$ for vehicle velocity of $80 \mathrm{~m} / \mathrm{s}$ and beam damping ratios of $0,0.025$ and 0.05 . Table 2 shows the results of the present solution using meshes of different fineness, while Table 3 shows those by CSEM using different numbers of modes. Convergence is studied by comparison with their respective most accurate results. They show that damping has little effect on the rate of convergence.

Fig. 17 compares the dynamic magnification factors $D_{\mathrm{M} 1}, D_{\mathrm{M} 2}$ and $D_{\mathrm{M} 3}$ obtained by the proposed formula with 8 elements versus velocity. As $D_{\mathrm{M} 3}$ never exceeds $D_{\mathrm{M} 1}$ for the same velocity, the use of dynamic magnification factor $D_{\mathrm{M} 3}$ (i.e. based on mid-span) for design is inadequate. Secondly, for the same velocity, $D_{\mathrm{M} 1}$ and $D_{\mathrm{M} 2}$ are the same, which means that the maximum value of $M(x, t)$ occurs at the sections beneath the four wheelsets. Therefore, if the dynamic magnification factor for bending moment is of interest, one may calculate $D_{\mathrm{M} 2}$ 
instead of $D_{\mathrm{M} 1}$, which will be much faster. For example, to compute $D_{\mathrm{M} 1}, D_{\mathrm{M} 2}$ and $D_{\mathrm{M} 3}$ in this case needs $45383 \mathrm{~s}$, while it only takes $326.5 \mathrm{~s}$ to compute $D_{\mathrm{M} 2}$ and $D_{\mathrm{M} 3}$.

Some of the parameters are then varied to evaluate their effects on the dynamic magnification factors $D_{\mathrm{M} 1}, D_{\mathrm{M} 2}$ and $D_{\mathrm{M} 3}$. As opposed to the case shown in Table 1 with a small mass ratio of the vehicle to the beam of about $5 \%$, a hypothetical case of a large mass ratio of about 0.795 is studied with $\bar{m}_{\mathrm{b}}=2303 \mathrm{~kg} / \mathrm{m}$ and $I_{\mathrm{b}}=2.9 \mathrm{~m}^{4}$ with the results shown in Fig. 18. Figs. 19 and 20 show respectively the results of two other cases of span lengths of 20 $\mathrm{m}$ and $40 \mathrm{~m}$, while the other parameters are from Table 1. Figs. 18 to 20 all show that the previous conclusions related to the dynamic magnification factors are still valid.

Because of the presence of top surface irregularities, Coriolis and centripetal inertial forces are induced [24]. The Coriolis and centripetal effects are associated with the appropriate terms which contain $v$ or $v^{2}$ in matrices $\mathbf{K}_{\mathrm{b} 2}, \mathbf{C}_{\mathrm{b} 2}$ and $\mathbf{K}_{\mathrm{v}_{j} \mathrm{~b}_{i}}$, vectors $\mathbf{F}_{\mathrm{v} j}$ and $\mathbf{F}_{\mathrm{bhj}}, \quad \dot{y}_{\mathrm{whj}}(t)$ and $\ddot{y}_{\mathrm{whj}}(t)$. The following cases are used to evaluate their effects on the dynamic magnification factors $D_{\mathrm{M} 1}$ and $D_{\mathrm{M} 3}$ :

(a) Case 1: Without beam irregularities; Coriolis / centripetal effects neglected

(b) Case 2: Without beam irregularities; Coriolis / centripetal effects included

(c) Case 3: With beam irregularities; Coriolis / centripetal effects neglected

(d) Case 4: With beam irregularities; Coriolis / centripetal effects included

For those cases with beam irregularities, an isolated irregularity given in terms of the length abscissa $x$ as $r(x)=\widetilde{a}\left(1-\cos 2 \pi x / l_{a}\right) / 2$ with a maximum depth $\widetilde{a}=1.5 \mathrm{~mm}$ and length $l_{a}=4 \mathrm{~m}$ is symmetrically located at mid-span. Figs. 21 and 22 show, respectively, the dynamic magnification factors $D_{\mathrm{M} 1}$ and $D_{\mathrm{M} 3}$ versus velocity. For the cases without irregularities, the Coriolis and centripetal effects are negligible as the slope and curvature of deflection of the stiff beam are small and hence there is little effect on the forces $P_{\text {whj }}$ in Eq. (19). However, for the cases with irregularities, $D_{\mathrm{M} 1}$ and $D_{\mathrm{M} 3}$ are underestimated when the 
Coriolis and centripetal effects are neglected, as the irregularities, and the Coriolis and centripetal forces have significant effects on the forces $P_{\mathrm{whj}}$ in Eq. (19).

\subsection{Example 3. A three-span continuous beam under a moving train}

Consider a three-span continuous beam $(3 \times 30 \mathrm{~m})$ under a moving train comprising three identical four-wheelset vehicles at centre-to-centre spacing of $25 \mathrm{~m}$ with the parameters given in Table 1. The case with train velocity of $50 \mathrm{~m} / \mathrm{s}$ is analysed by the proposed formulae and the conventional finite element formulae with 12 elements of equal lengths. It is assumed that the beam is at rest at time $t=0 \mathrm{~s}$ when the first wheelset of the first vehicle runs onto the left end of the beam. Figs. 23 and 24 show the internal force distributions along the beam at time $t$ $=1.5 \mathrm{~s}$ when the first wheelset of the first vehicle is at the middle of the right end span. The present method can accurately capture the kinks in bending moment and the discontinuities in shear force, which are associated with the positions of wheelsets. The results from the conventional finite element formulae are governed by the finite element mesh and thus erroneous. The present method is superior in correctly evaluating the shear forces around interior supports.

Then the train velocity is varied from $10 \mathrm{~m} / \mathrm{s}$ to $200 \mathrm{~m} / \mathrm{s}$ at $2.5 \mathrm{~m} / \mathrm{s}$ intervals for evaluation of the dynamic magnification factors using 24 elements of equal lengths. For calculation of $D_{\mathrm{M} 1}, D_{\mathrm{M} 2}$ and $D_{\mathrm{M} 3}$ here using Eq. (27), $M_{2}$ is taken as the maximum dynamic bending moment of the beam sections beneath all wheelsets for the velocity, while $M_{\text {st }}$ is taken as the maximum static bending moment of the central mid-span beam section. The dynamic magnification factors shown in Fig. 25 confirm the conclusions drawn in Example 2. Again for the same velocity, $D_{\mathrm{M} 3}$ never exceeds $D_{\mathrm{M} 1}$, and $D_{\mathrm{M} 1}$ and $D_{\mathrm{M} 2}$ are the same.

\section{Conclusions}

Based on the conditions of dynamic equilibrium, this paper presents new finite-element formulae for calculating the internal forces at any arbitrary section of Bernoulli-Euler beams 
under moving vehicles. Apart from verifying the correctness of the proposed formulae, the following conclusions can be drawn.

(a) The proposed formulae are much more accurate than the conventional finite element formulae in the evaluation of internal forces of beam, and the accuracy of the proposed formulae is better than that of CSEM in the evaluation of the internal forces of beam, especially for shear force.

(b) The use of dynamic magnification factor $D_{\mathrm{M} 3}$ for bending moment at mid-span beam section for the design is inadequate, as it often underestimates the bending moment due to moving loads.

(c) From the numerical examples of simply supported and continuous beams under a moving force or vehicle, there is a tendency for the dynamic magnification factor $D_{\mathrm{M} 2}$ for bending moment at the beam section beneath moving force and the dynamic magnification factor $D_{\mathrm{M} 1}$ for bending moment at all beam sections to be the same in the lower range of velocity. Therefore one may use $D_{\mathrm{M} 2}$ instead of $D_{\mathrm{M} 1}$ in the lower range of velocity commonly encountered.

\section{Acknowledgements}

The work was supported by the National Natural Science Foundation of China (Grants 51078360 and 50938008), and Hunan Provincial Natural Science Foundation of China (Grant 10JJ3059).

\section{References}

[1] L. Frýba, Dynamics of Railway Bridges, Thomas Telford, London, 1996.

[2] H.S. Tzou, L.A. Bergman, Dynamics and Control of Distributed Systems, Cambridge University Press, Cambridge, 1998.

[3] L. Frýba, Vibrations of Solids and Structures Under Moving Loads, Thomas Telford, London, 1999. 
[4] A.V. Pesterev, L.A. Bergman, An improved series expansion of the solution to the moving oscillator problem. Journal of Vibration and Acoustics 122(2000) 54-61.

[5] A.V. Pesterev, C.A. Tan, L.A. Bergman, A new method for calculating bending moment and shear force in moving load problems. Journal of Applied Mechanics 68(2001) 252-259.

[6] B. Biondi, G. Muscolino, A. Sidoti, Methods for calculating bending moment and shear force in the moving mass problem. Journal of Vibration and Acoustics 126(2004) 542-552.

[7] B. Biondi, G. Muscolino, New improved series expansion for solving the moving oscillator problem. Journal of Sound and Vibration 281(2005) 99-117.

[8] C. Bilello, D.M. Paola, S. Salamone, A correction method for the analysis of continuous linear one-dimensional systems under moving loads. Journal of Sound and Vibration $315(2008) 226-238$.

[9] P. Lou, X.G. Zhong, J.F. Tang, Q.Y. Zeng, Finite-element analysis of discretely supported rail subjected to multiple moving concentrated forces. Proceedings of the Institution of Mechanical Engineers Part F: Journal of Rail and Rapid Transit 220(2006) 305-315.

[10] P. Lou, Finite-element formulae for calculating the sectional forces of a Bernoulli-Euler beam on continuously viscoelastic foundation subjected to moving concentrated loads. Shock and Vibration 15(2008) 147-162.

[11] European Rail Research Institute (ERRI) D-214 Committee. Rail bridges for speeds $>$ 200 km/h. RP 9, Final report. Utretch, The Netherlands, 1999.

[12] Y.S. Cheng, F.T.K. Au, Y.K. Cheung, Vibration of railway bridges under a moving train by using bridge-track-vehicle element. Engineering Structures 23(2001) 1597-1606.

[13] Y.S. Wu, Y.B. Yang, Steady-state response and riding comfort of trains moving over a series of simply supported bridges. Engineering Structures 25(2003) 251-265.

[14] Y.B. Yang, Y.S. Wu, A versatile element for analyzing vehicle-bridge interaction response. Engineering Structures 23(2001) 452-469.

[15] P. Lou, Finite element analysis for train-track-bridge interaction system. Archive of Applied Mechanics 77(2007) 707-728. 
[16] F.T.K. Au, J.J. Wang, Y.K. Cheung, Impact study of cable-stayed bridge under railway traffic using various models. Journal of Sound and Vibration 240(2001) 447-465.

[17] K. J. Bathe, E. L. Wilson, Numerical Methods in Finite Element Analysis, Prentice-Hall Inc., Englewood Cliffs, N.J., 1976.

[18] M. Paz, Structural Dynamics: Theory and Computation, fourth ed., Chapman \& Hall, New York, 1997.

[19] J.L. Humar, Dynamics of Structures, $2^{\text {nd }}$ ed., A.A. Balkema Publishers, 2002.

[20] G.B. Warburton, The Dynamical Behaviour of Structures, $2^{\text {nd }}$ ed., Pergamon Press, Oxford, 1976.

[21] S. Timoshenko, D.H. Young, W. Weaver Jr., Vibration Problems in Engineering, 4th Edition, Wiley, New York, 1974.

[22] J.M. Biggs, Introduction to Structural Dynamics, McGraw-Hill, Inc., New York, 1964.

[23] P. Lou, G.L. Dai, Q.Y. Zeng, Modal coordinate formulations for a simply supported bridge subjected to a moving train modelled as two-stage suspension vehicles. Proceedings of the Institution of Mechanical Engineers Part C: Journal of Mechanical Engineering Science 219(2005), 1027-1040.

[24] G.T. Michaltsos, The influence of centripetal and Coriolis forces on the dynamic response of light bridges under moving vehicles. Journal of Sound and Vibration 247(2001) 261-277. 


\section{Figure captions}

Fig. 1. A train running on a multi-span continuous railway bridge

Fig. 2. A typical vehicle running on the $i$-th span continuous beam

Fig. 3. Generalized nodal forces $Q_{1}^{\mathrm{e}}, M_{1}^{\mathrm{e}}, Q_{\mathrm{r}}^{\mathrm{e}}$ and $M_{\mathrm{r}}^{\mathrm{e}}$ of a typical beam element

Fig. 4. Free-body diagram for calculating the shear force $Q_{\mathrm{A}}$ and bending moment $M_{\mathrm{A}}$ at point A where $f_{\mathrm{I}}(\xi)=\bar{m}_{\mathrm{b}} \mathbf{N} \ddot{\mathbf{q}}^{\mathrm{e}}$

Fig. 5. Example 1: Comparison of dynamic magnification factor $D_{\mathrm{M} 1}$ versus velocity parameter $\psi$

Fig. 6. Example 1: Comparison of dynamic magnification factor $D_{\mathrm{M} 2}$ versus velocity parameter $\psi$

Fig. 7. Example 1: Comparison of dynamic magnification factor $D_{\mathrm{M} 3}$ versus velocity parameter $\psi$

Fig. 8. Example 1: Dynamic magnification factors $D_{\mathrm{M} 1}, D_{\mathrm{M} 2}$ and $D_{\mathrm{M} 3}$ obtained by the proposed formula with 8 elements versus velocity parameter $\psi$

Fig. 9. Example 1: Comparison of shear force distribution along the beam at $t=0.24 \mathrm{~s}$

Fig. 10. Example 2: Comparison of shear force distribution along the beam at $t=0.48 \mathrm{~s}$

Fig. 11. Example 2: Comparison of bending moment distributions along the beam at $t=0.48 \mathrm{~s}$

Fig. 12. Example 2: Convergence of shear force distribution along the beam at $t=0.48 \mathrm{~s}$ by

\section{CSEM}

Fig. 13. Example 2: Convergence of bending moment distribution along the beam at $t=0.48 \mathrm{~s}$ by CSEM

Fig. 14. Example 2: Comparison of dynamic magnification factor $D_{\mathrm{M} 1}$ versus velocity

Fig. 15. Example 2: Comparison of dynamic magnification factor $D_{\mathrm{M} 2}$ versus velocity

Fig. 16. Example 2: Comparison of dynamic magnification factor $D_{\mathrm{M} 3}$ versus velocity 
Fig. 17. Example 2: Dynamic magnification factors $D_{\mathrm{M} 1}, D_{\mathrm{M} 2}$ and $D_{\mathrm{M} 3}$ obtained by the proposed formula with 8 elements versus velocity

Fig. 18. Example 2: Dynamic magnification factors $D_{\mathrm{M} 1}, D_{\mathrm{M} 2}$ and $D_{\mathrm{M} 3}$ obtained by the proposed formula with 8 elements versus velocity (hypothetical case of large mass ratio with $\bar{m}_{\mathrm{b}}=2,303 \mathrm{~kg} / \mathrm{m}$ and $\left.I_{\mathrm{b}}=2.9 \mathrm{~m}^{4}\right)$

Fig. 19. Example 2: Dynamic magnification factors $D_{\mathrm{M} 1}, D_{\mathrm{M} 2}$ and $D_{\mathrm{M} 3}$ obtained by the proposed formula with 8 elements versus velocity (span length $=20 \mathrm{~m}$ )

Fig. 20. Example 2: Dynamic magnification factors $D_{\mathrm{M} 1}, D_{\mathrm{M} 2}$ and $D_{\mathrm{M} 3}$ obtained by the proposed formula with 8 elements versus velocity (span length $=40 \mathrm{~m}$ )

Fig. 21. Example 2: Effects of irregularities, Coriolis force and centripetal force on dynamic magnification factor $D_{\mathrm{M} 1}$

Fig. 22. Example 2: Effects of irregularities, Coriolis force and centripetal force on dynamic magnification factor $D_{\mathrm{M} 3}$

Fig. 23. Example 3: Comparison of bending moment distribution along the beam at $t=1.5 \mathrm{~s}$

Fig. 24. Example 3: Comparison of shear force distribution along the beam at $t=1.5 \mathrm{~s}$

Fig. 25. Example 3: Dynamic magnification factors $D_{\mathrm{M} 1}, D_{\mathrm{M} 2}$ and $D_{\mathrm{M} 3}$ obtained by the proposed formula with 24 elements versus velocity 


\section{Figures}

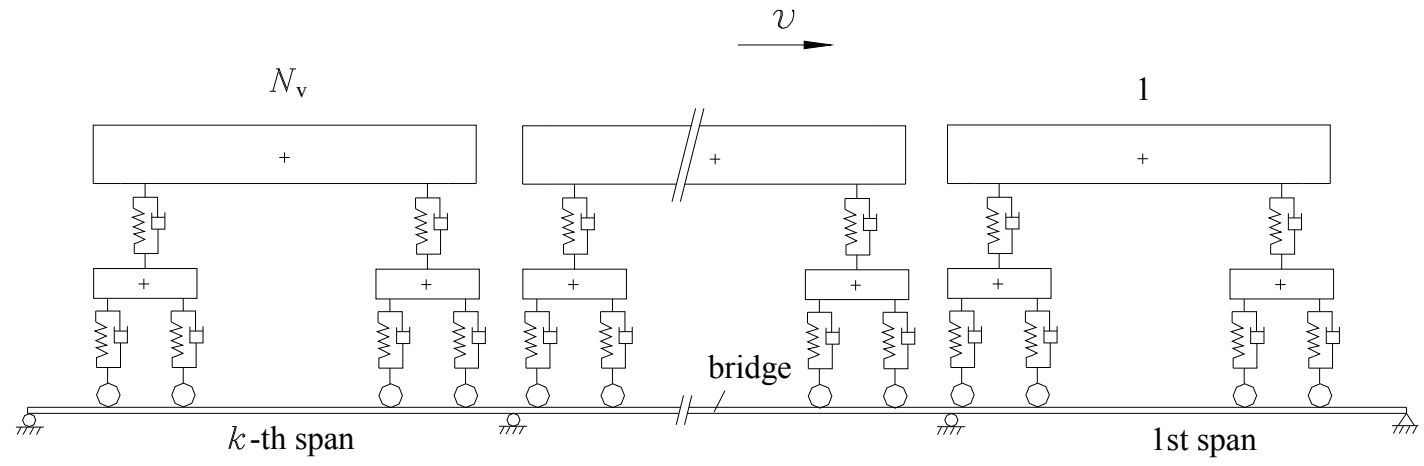

Fig. 1. A train running on a multi-span continuous railway bridge

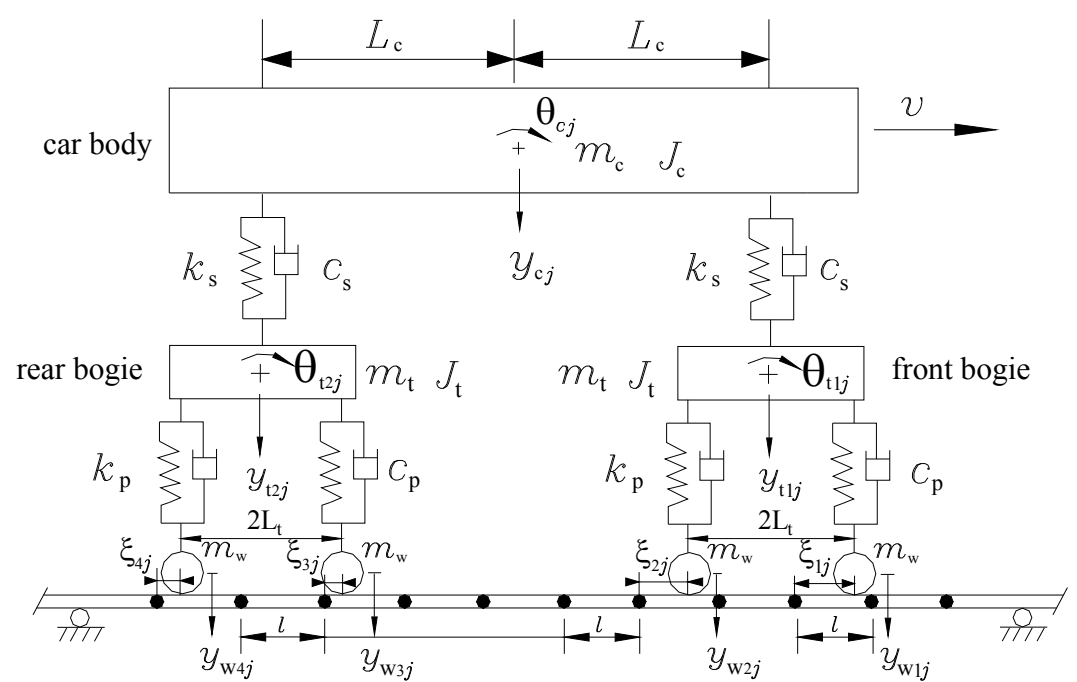

Fig. 2. A typical vehicle running on the $i$-th span continuous beam

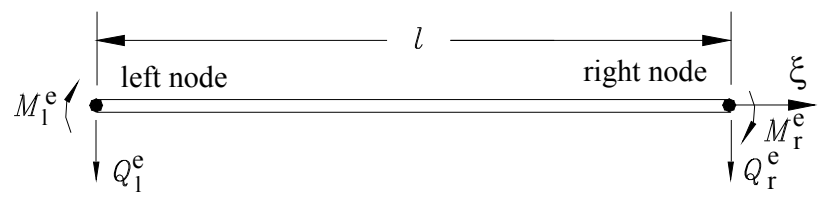

Fig. 3. Generalized nodal forces $Q_{1}^{\mathrm{e}}, M_{1}^{\mathrm{e}}, Q_{\mathrm{r}}^{\mathrm{e}}$ and $M_{\mathrm{r}}^{\mathrm{e}}$ of a typical beam element 


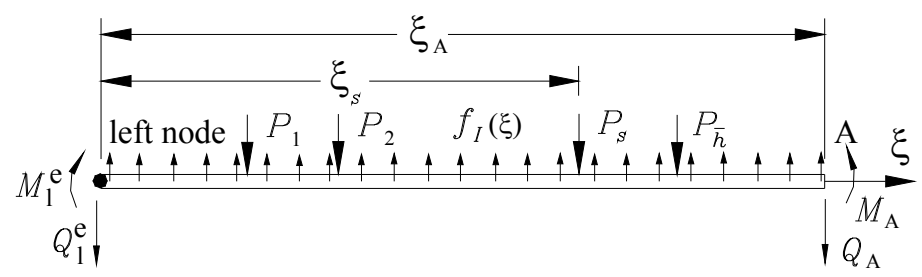

Fig. 4. Free-body diagram for calculating the shear force $Q_{\mathrm{A}}$ and bending moment $M_{\mathrm{A}}$ at point A where $f_{\mathrm{I}}(\xi)=\bar{m}_{\mathrm{b}} \mathbf{N} \ddot{\mathbf{q}}^{\mathrm{e}}$

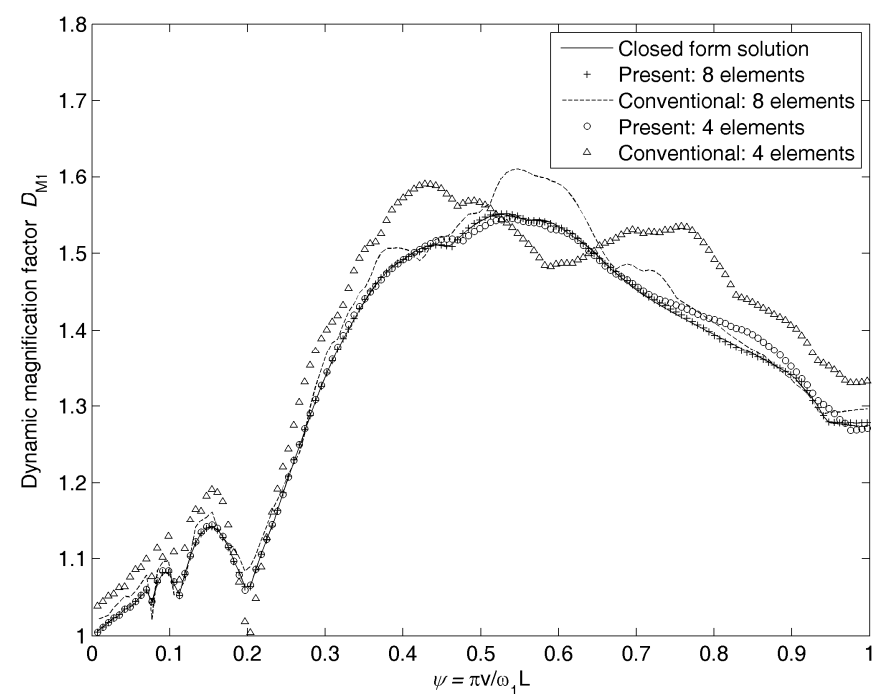

Fig. 5. Example 1: Comparison of dynamic magnification factor $D_{\mathrm{M} 1}$ versus velocity parameter $\psi$

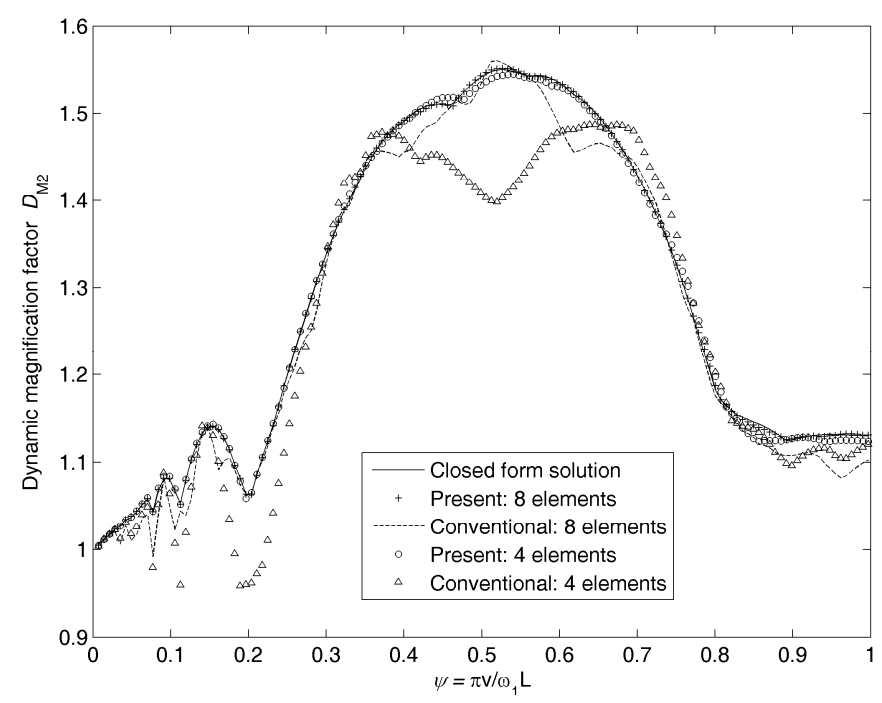

Fig. 6. Example 1: Comparison of dynamic magnification factor $D_{\mathrm{M} 2}$ versus velocity parameter $\psi$ 


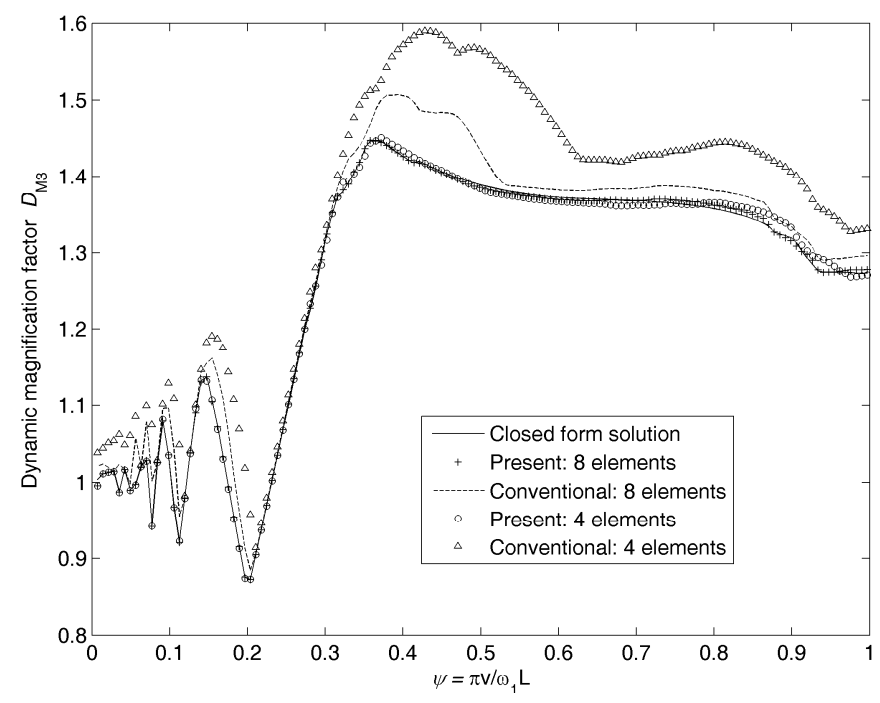

Fig. 7. Example 1: Comparison of dynamic magnification factor $D_{\mathrm{M} 3}$ versus velocity parameter $\psi$

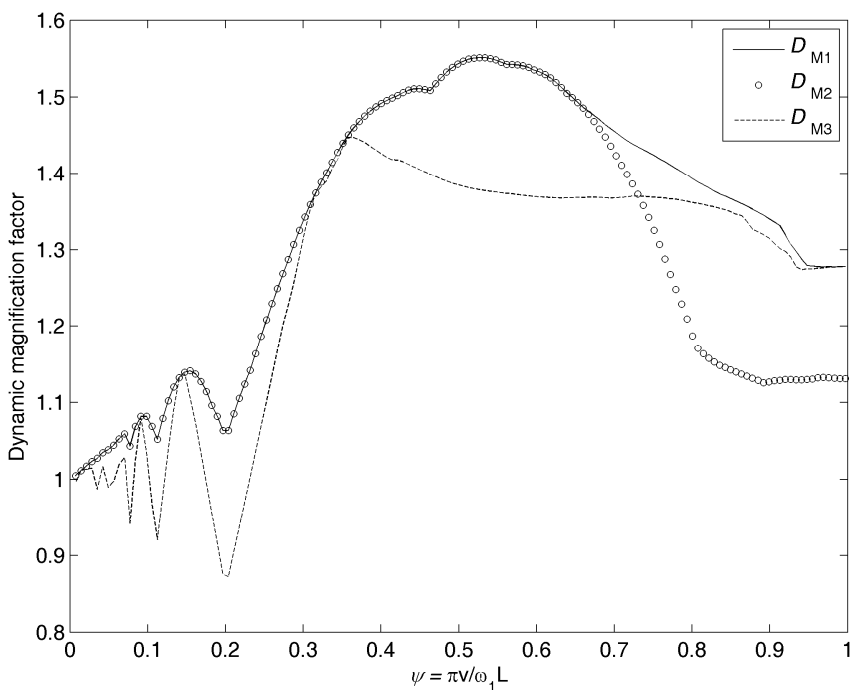

Fig. 8. Example 1: Dynamic magnification factors $D_{\mathrm{M} 1}, D_{\mathrm{M} 2}$ and $D_{\mathrm{M} 3}$ obtained by the proposed formula with 8 elements versus velocity parameter $\psi$ 


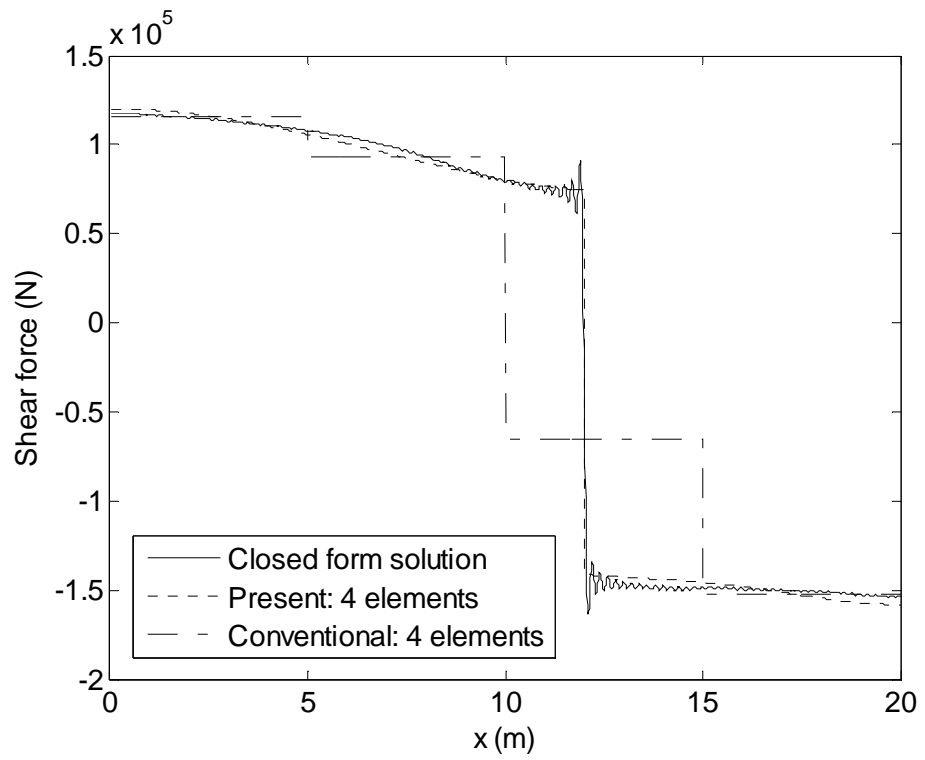

Fig. 9. Example 1: Comparison of shear force distribution along the beam at $t=0.24 \mathrm{~s}$

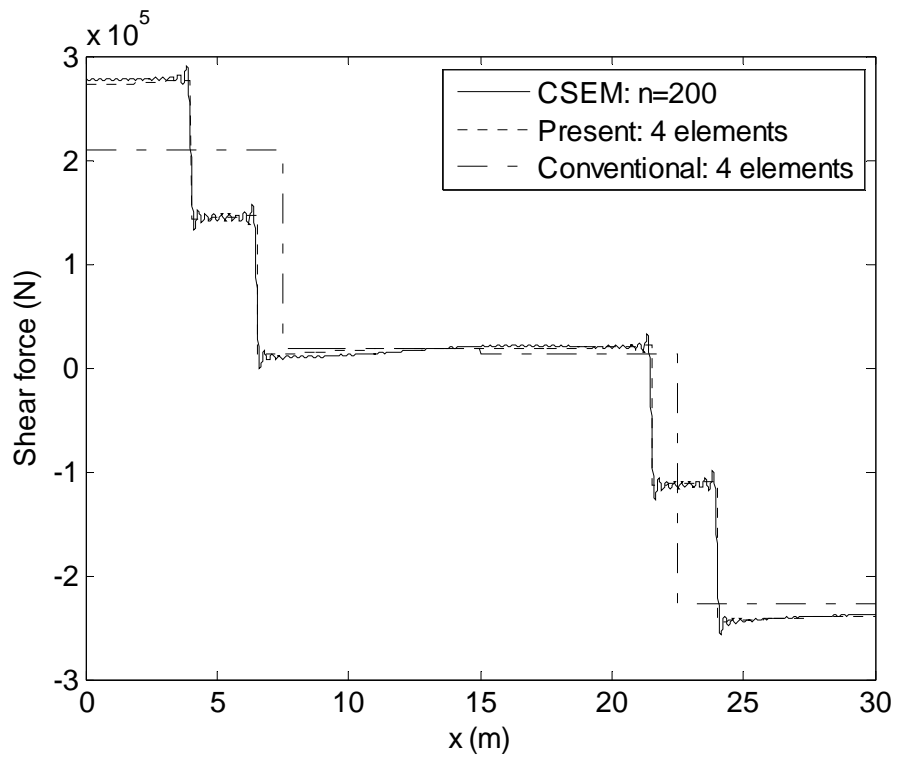

Fig. 10. Example 2: Comparison of shear force distribution along the beam at $t=0.48 \mathrm{~s}$ 


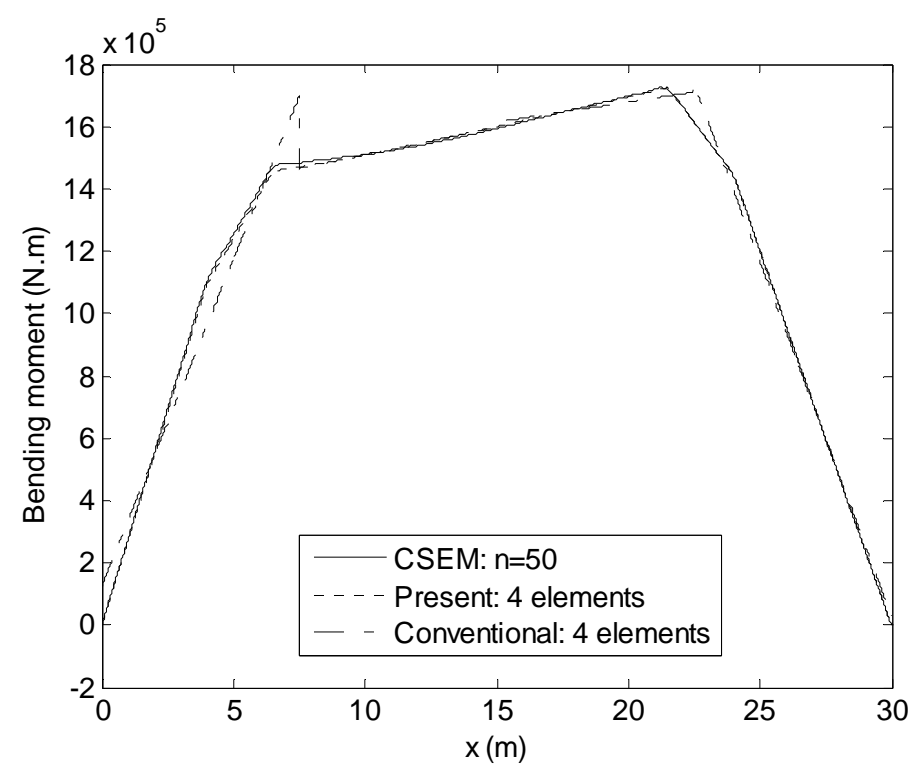

Fig. 11. Example 2: Comparison of bending moment distribution along the beam at $t=0.48 \mathrm{~s}$

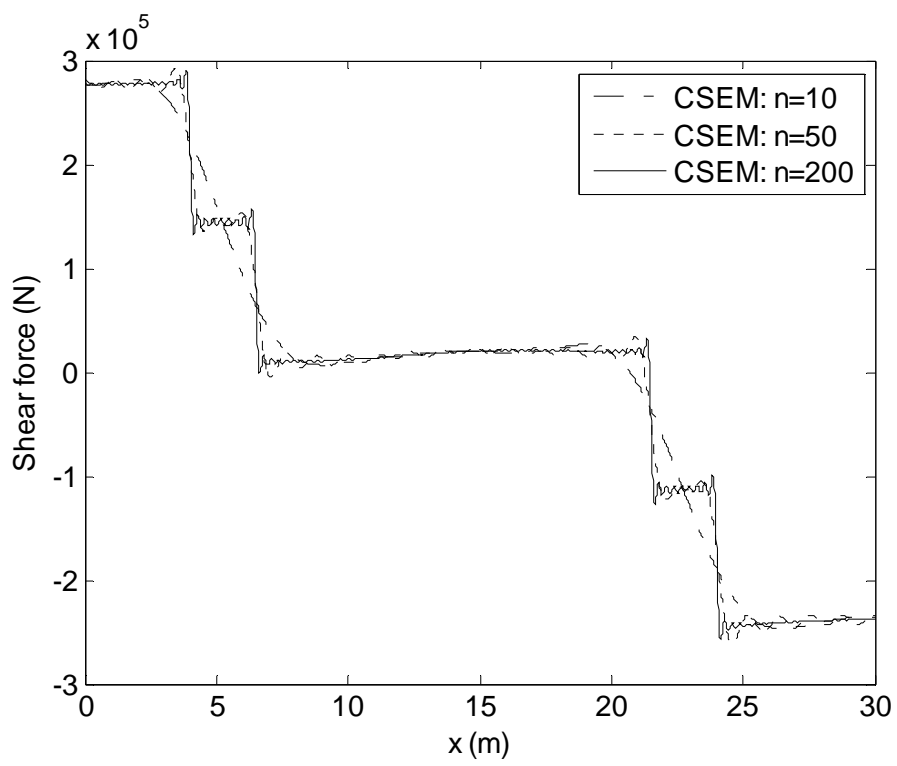

Fig. 12. Example 2: Convergence of shear force distribution along the beam at $t=0.48 \mathrm{~s}$ by CSEM 


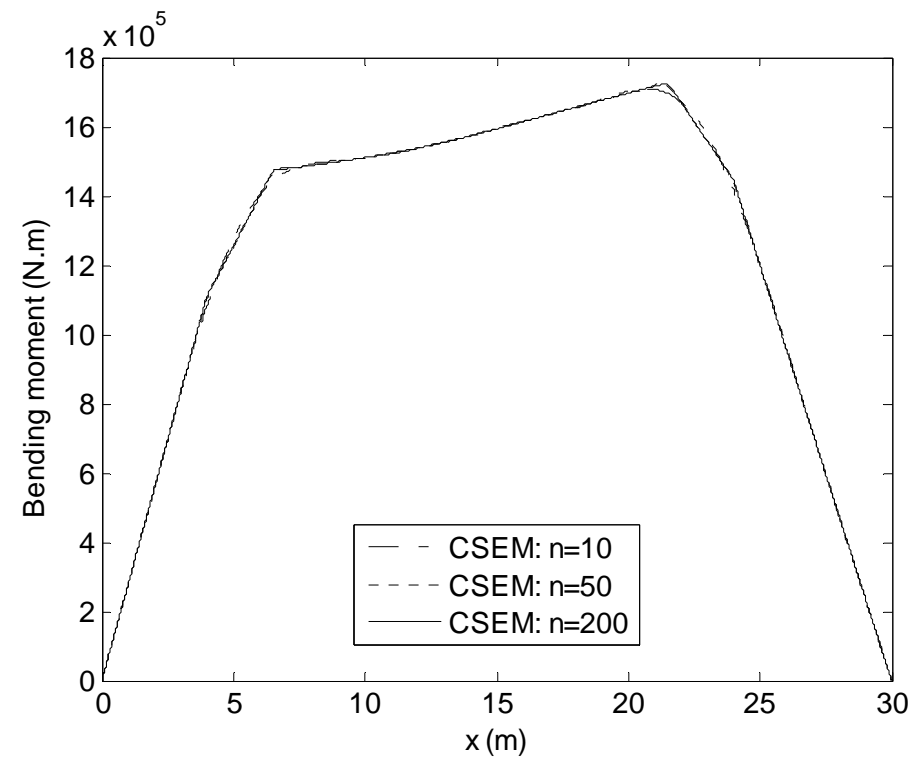

Fig. 13. Example 2: Convergence of bending moment distribution along the beam at $t=0.48 \mathrm{~s}$ by CSEM

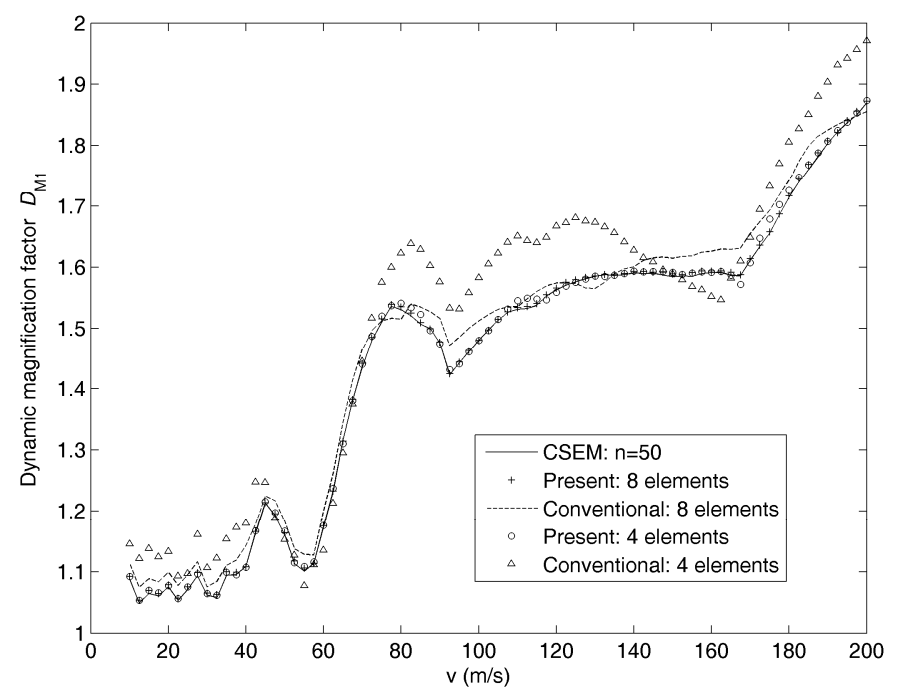

Fig. 14. Example 2: Comparison of dynamic magnification factor $D_{\mathrm{M} 1}$ versus velocity 


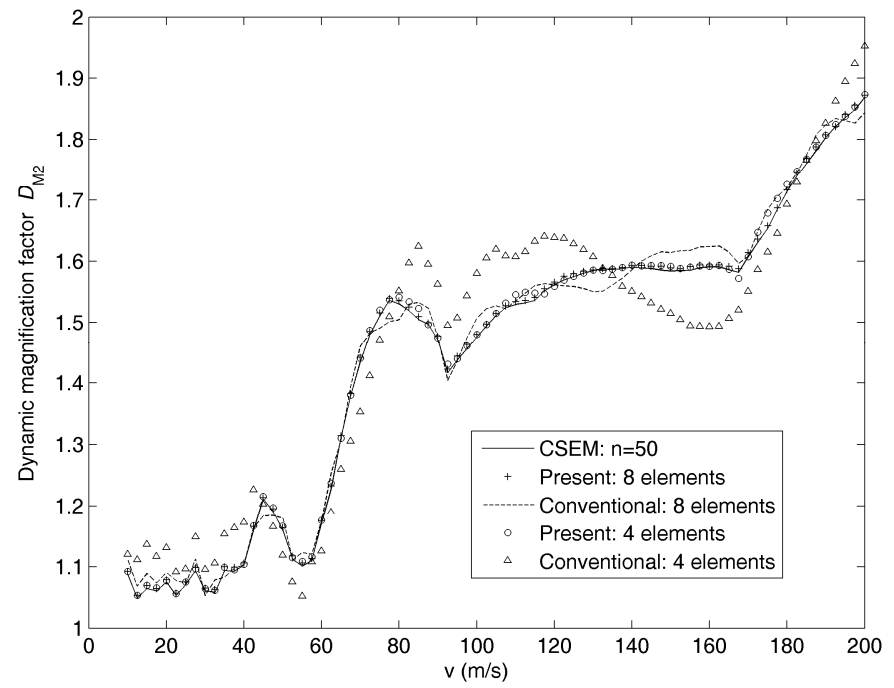

Fig. 15. Example 2: Comparison of dynamic magnification factor $D_{\mathrm{M} 2}$ versus velocity

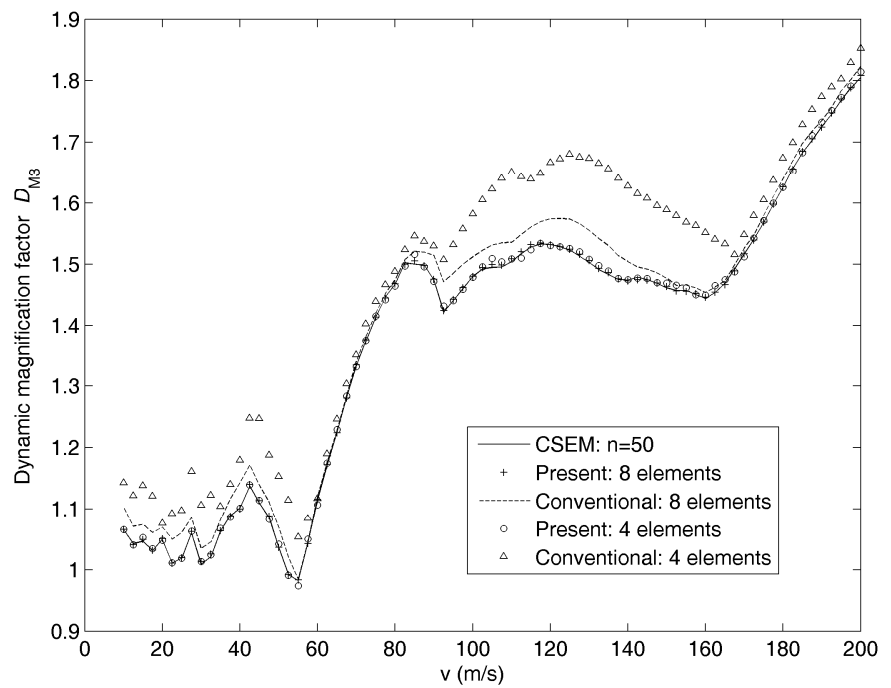

Fig. 16. Example 2: Comparison of dynamic magnification factor $D_{\mathrm{M} 3}$ versus velocity 


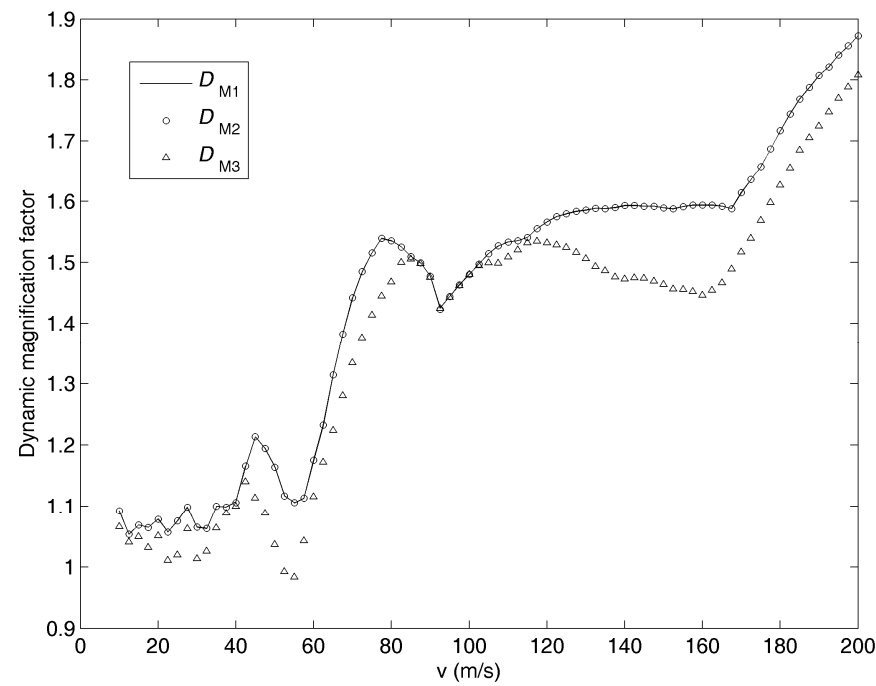

Fig. 17. Example 2: Dynamic magnification factors $D_{\mathrm{M} 1}, D_{\mathrm{M} 2}$ and $D_{\mathrm{M} 3}$ obtained by the proposed formula with 8 elements versus velocity

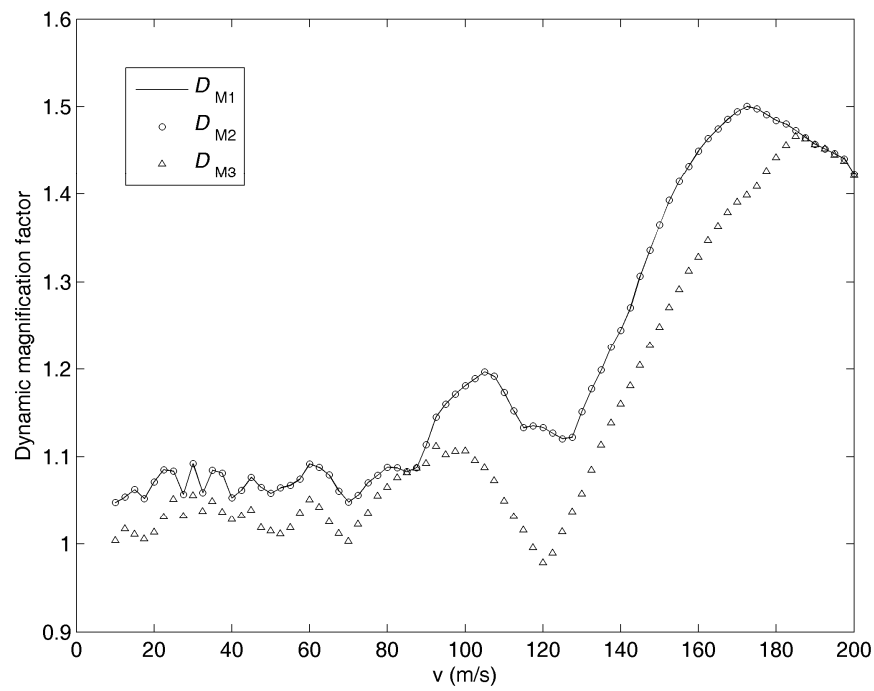

Fig. 18. Example 2: Dynamic magnification factors $D_{\mathrm{M} 1}, D_{\mathrm{M} 2}$ and $D_{\mathrm{M} 3}$ obtained by the proposed formula with 8 elements versus velocity (hypothetical case of large mass ratio with $\bar{m}_{\mathrm{b}}=2,303 \mathrm{~kg} / \mathrm{m}$ and $I_{\mathrm{b}}=2.9 \mathrm{~m}^{4}$ ) 


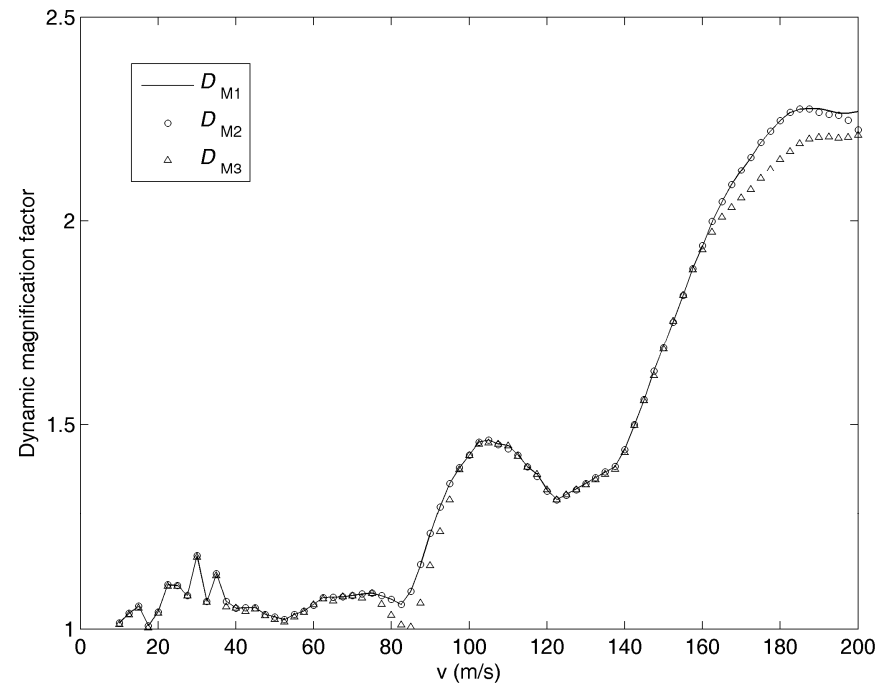

Fig. 19. Example 2: Dynamic magnification factors $D_{\mathrm{M} 1}, D_{\mathrm{M} 2}$ and $D_{\mathrm{M} 3}$ obtained by the proposed formula with 8 elements versus velocity ( span length $=20 \mathrm{~m}$ )

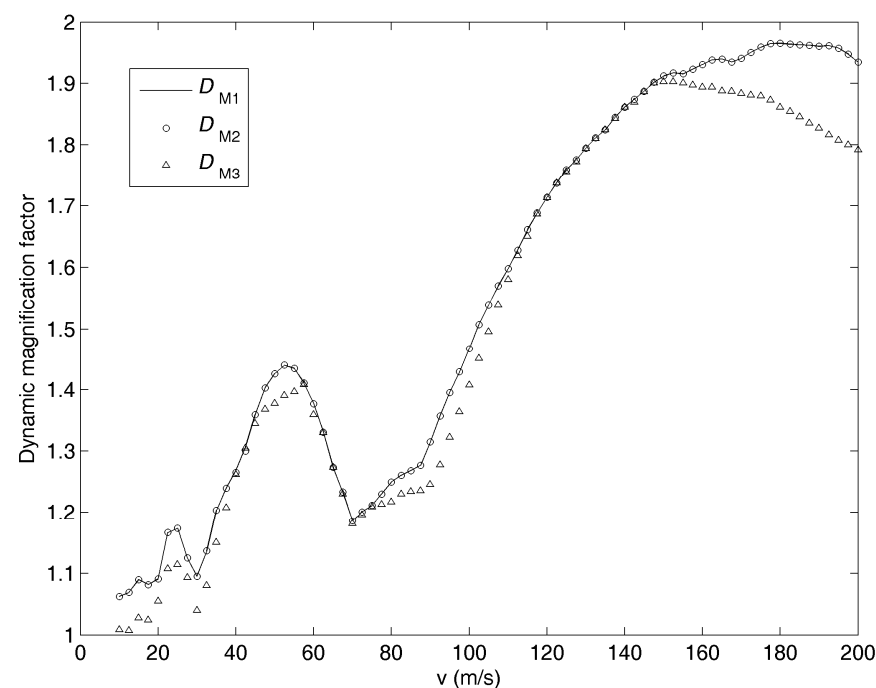

Fig. 20. Example 2: Dynamic magnification factors $D_{\mathrm{M} 1}, D_{\mathrm{M} 2}$ and $D_{\mathrm{M} 3}$ obtained by the proposed formula with 8 elements versus velocity $($ span length $=40 \mathrm{~m})$ 


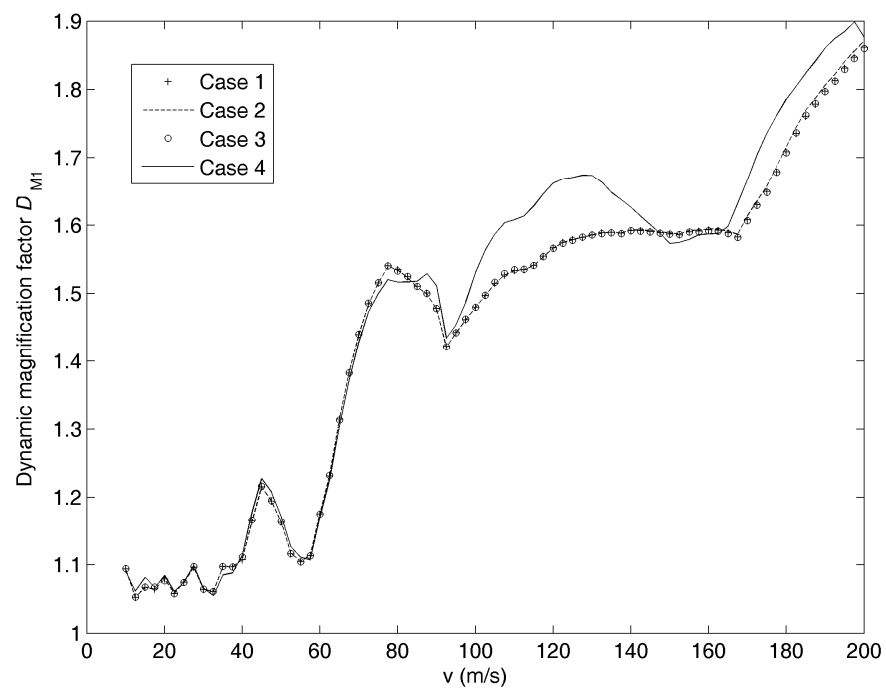

Fig. 21. Example 2: Effects of irregularities, Coriolis force and centripetal force on dynamic magnification factor $D_{\mathrm{M} 1}$

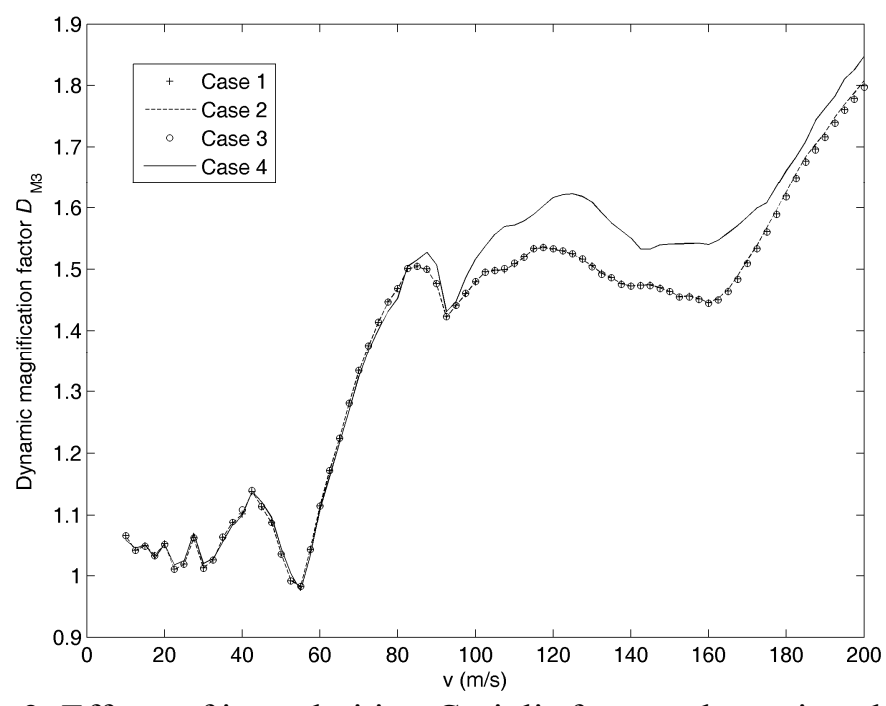

Fig. 22. Example 2: Effects of irregularities, Coriolis force and centripetal force on dynamic magnification factor $D_{\mathrm{M} 3}$

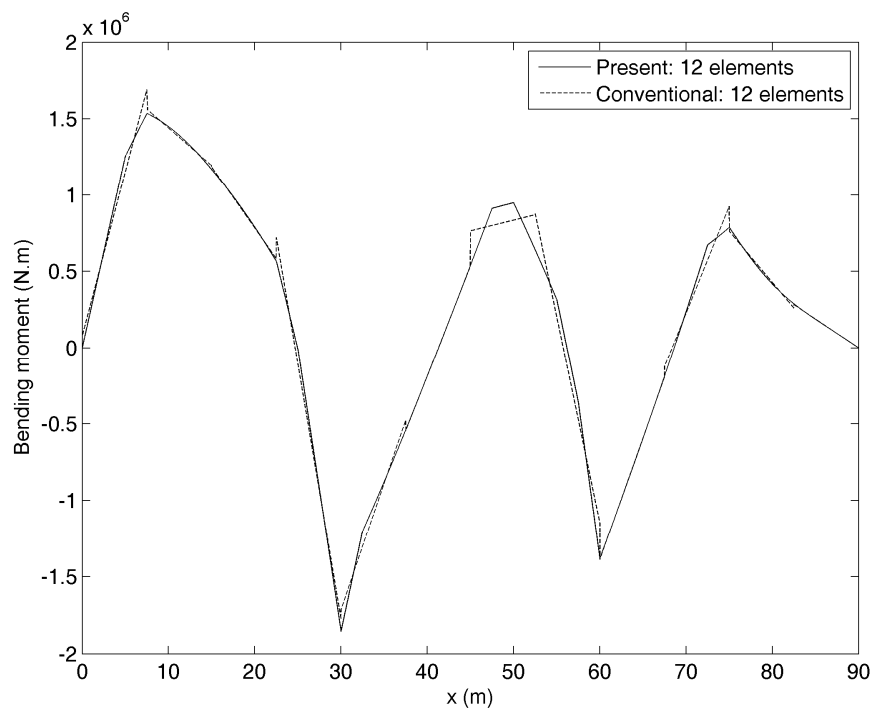


Fig. 23. Example 3: Comparison of bending moment distribution along the beam at $t=1.5 \mathrm{~s}$

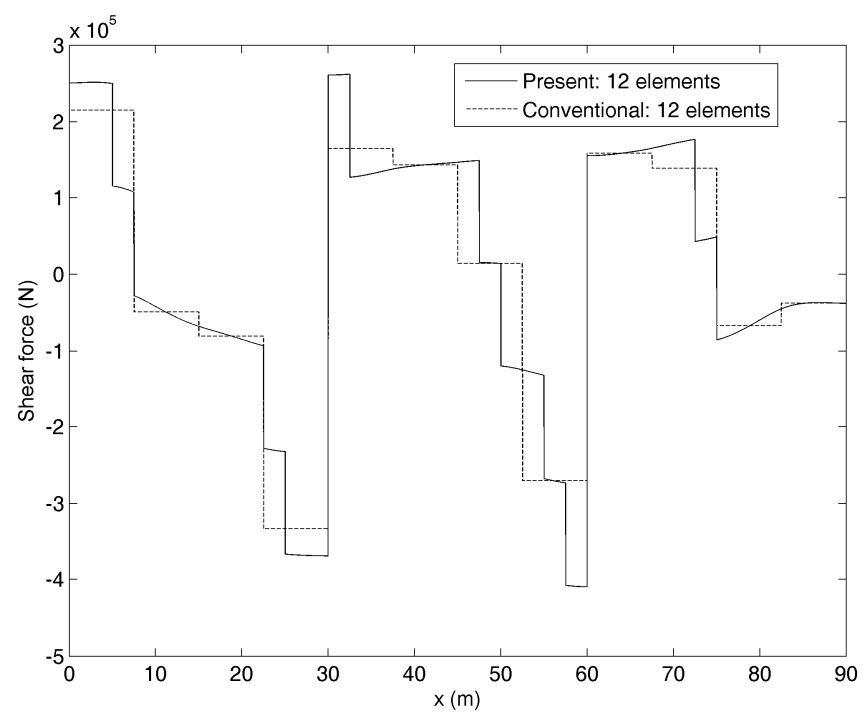

Fig. 24. Example 3: Comparison of shear force distribution along the beam at $t=1.5 \mathrm{~s}$

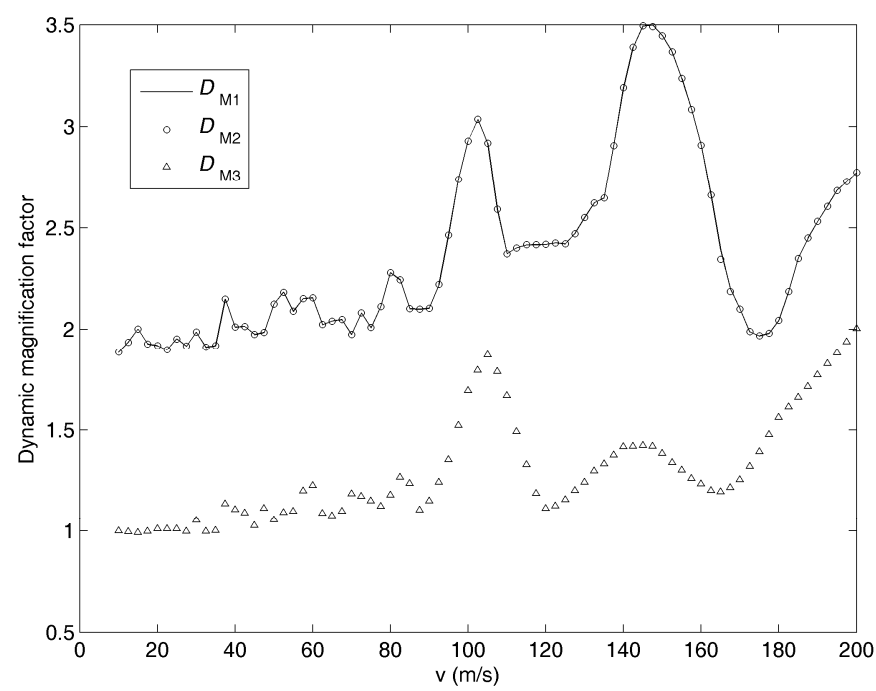

Fig. 25. Example 3: Dynamic magnification factors $D_{\mathrm{M} 1}, D_{\mathrm{M} 2}$ and $D_{\mathrm{M} 3}$ obtained by the proposed formula with 24 elements versus velocity 


\section{Tables}

Table 1. Properties of vehicle and beam for Examples 2 and 3

\begin{tabular}{lll}
\hline Notation & Item & Value \\
\hline Vehicle & Mass of car body & $4.175 \times 10^{4} \mathrm{~kg}$ \\
$m_{\mathrm{c}}$ & Mass moment of inertia of car body & $2.08 \times 10^{6} \mathrm{~kg} \cdot \mathrm{m}^{2}$ \\
$J_{\mathrm{c}}$ & Spring stiffness of the second suspension system & $5.3 \times 10^{5} \mathrm{~N} / \mathrm{m}$ \\
$k_{\mathrm{s}}$ & Damping coefficient of the second suspension system & $9.02 \times 10^{4} \mathrm{~N} \cdot \mathrm{s} / \mathrm{m}$ \\
$c_{\mathrm{s}}$ & Half of longitudinal distance between the centre of gravity of front & $8.75 \mathrm{~m}$ \\
$L_{\mathrm{c}}$ & bogie and of rear bogie & \\
$m_{\mathrm{t}}$ & Mass of a bogie frame & $3.04 \times 10^{3} \mathrm{~kg}$ \\
$J_{\mathrm{t}}$ & Mass moment of inertia of a bogie frame & $3.93 \times 10^{3} \mathrm{~kg} \cdot \mathrm{m}^{2}$ \\
$L_{\mathrm{t}}$ & Half of bogie axle base & $1.25 \mathrm{~m}$ \\
$k_{\mathrm{p}}$ & Spring stiffness of the primary suspension system & $1.18 \times 10^{6} \mathrm{~N} / \mathrm{m}$ \\
$C_{\mathrm{p}}$ & Damping coefficient of the primary suspension system & $3.92 \times 10^{4} \mathrm{~N} \cdot \mathrm{s} / \mathrm{m}$ \\
$m_{\mathrm{w}}$ & Mass of a wheelset & $1.78 \times 10^{3} \mathrm{~kg}$ \\
$l_{\mathrm{s}}$ & Longitudinal distance between the centre of gravity of bogie and & $3.75 \mathrm{~m}$ \\
& nearest side of vehicle body & \\
Beam & $\quad$ Span length & \\
$L$ & Young's modulus & $30 \mathrm{~m}$ \\
$E_{\mathrm{b}}$ & Moment of inertia & $2.943 \times 10^{10} \mathrm{~Pa}$ \\
$I_{\mathrm{b}}$ & Damping ratio & $8.65 \mathrm{~m}{ }^{4}$ \\
$\zeta$ & Mass per unit length & 0 \\
$\bar{m}_{\mathrm{b}}$ & & $3.6 \times 10^{4} \mathrm{~kg} / \mathrm{m}$ \\
\hline
\end{tabular}

Table 2. Example 2: Convergence of dynamic magnification factor $D_{\mathrm{M} 1}$ for different damping ratios $\zeta$ obtained by the proposed formula (vehicle velocity $=80 \mathrm{~m} / \mathrm{s}$ )

\begin{tabular}{cccc}
\hline \multirow{2}{*}{ Number of elements } & \multicolumn{3}{c}{ Dynamic magnification factor } \\
\cline { 2 - 4 } & $\zeta=0$ & $\zeta=0.025$ & $\zeta=0.05$ \\
\hline 4 & $1.541250(100.44 \%)$ & $1.464369(100.04 \%)$ & $1.405055(100.05 \%)$ \\
16 & $1.535184(100.05 \%)$ & $1.463653(99.99 \%)$ & $1.404264(99.99 \%)$ \\
32 & $1.534082(99.98 \%)$ & $1.463796(100.00 \%)$ & $1.404351(100.00 \%)$ \\
64 & $1.534393(99.98 \%)$ & $1.463788(100.00 \%)$ & $1.404346(100.00 \%)$ \\
& $1.534443(100.00 \%)$ & $1.463787(100.00 \%)$ & $1.404347(100.00 \%)$ \\
\hline
\end{tabular}

Table 3. Example 2: Convergence of dynamic magnification factor $D_{\mathrm{M} 1}$ for different damping ratios $\zeta$ obtained by CSEM (vehicle velocity $=80 \mathrm{~m} / \mathrm{s}$ )

\begin{tabular}{cccc}
\hline \multirow{2}{*}{ Number of modes } & \multicolumn{3}{c}{ Dynamic magnification factor } \\
\cline { 2 - 4 } & $\zeta=0$ & $\zeta=0.025$ & $\zeta=0.05$ \\
\hline 10 & $1.520650(99.19 \%)$ & $1.448848(99.09 \%)$ & $1.389911(99.12 \%)$ \\
18 & $1.522137(99.29 \%)$ & $1.450685(99.22 \%)$ & $1.391505(99.23 \%)$ \\
34 & $1.529613(99.77 \%)$ & $1.458613(99.76 \%)$ & $1.399159(99.77 \%)$ \\
66 & $1.531491(99.90 \%)$ & $1.460497(99.89 \%)$ & $1.400879(99.90 \%)$ \\
130 & $1.533074(100.00 \%)$ & $1.462142(100.00 \%)$ & $1.402320(100.00 \%)$ \\
\hline
\end{tabular}

